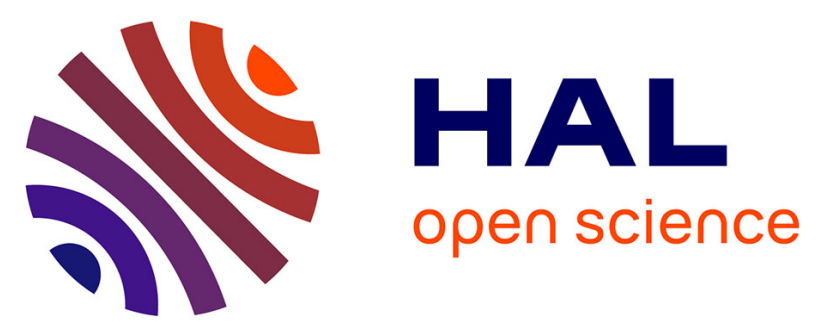

\title{
Émergence et affirmation d'une topographie chrétienne dans les villes de la Gaule méridionale Jean Guyon
}

\section{To cite this version:}

Jean Guyon. Émergence et affirmation d'une topographie chrétienne dans les villes de la Gaule méridionale. Gallia - Archéologie de la France antique, 2006, Antiquité tardive, haut Moyen Âge et premiers temps chrétiens en Gaule méridionale (première partie) : réseau des cités, monde urbain et monde des morts, 63, pp.85-110. 10.3406/galia.2006.3287 . hal-01914301

\section{HAL Id: hal-01914301 https://hal.science/hal-01914301}

Submitted on 8 Jan 2020

HAL is a multi-disciplinary open access archive for the deposit and dissemination of scientific research documents, whether they are published or not. The documents may come from teaching and research institutions in France or abroad, or from public or private research centers.
L'archive ouverte pluridisciplinaire HAL, est destinée au dépôt et à la diffusion de documents scientifiques de niveau recherche, publiés ou non, émanant des établissements d'enseignement et de recherche français ou étrangers, des laboratoires publics ou privés.

\section{(1) (1) $\$$}

Distributed under a Creative Commons Attribution - NonCommercial - NoDerivatives| 4.0 


\title{
ÉMERGENCE ET AFFIRMATION D'UNE TOPOGRAPHIE CHRÉTIENNE DANS LES VILLES DE LA GAULE MÉRIDIONALE
}

\author{
Jean GUYON
}

\begin{abstract}
Mots-clés. Groupe épiscopal, églises urbaines, églises funéraires, monastères.
Résumé. Dans le Midi, une à deux villes sur cinq comptaient dans l'Antiquité pour seul monument chrétien un groupe épiscopal et le bilan reste modeste, même pour les villes plus richement dotées. Hors les groupes épiscopaux, on compte en effet en moyenne deux à quatre édifices par ville, dont deux tiers environ étaient situés extra-muros et $20 \%$ répondaient à des monastères. À quoi s'ajoutent de fortes disparités régionales entre les deux Aquitaines et la Viennoise, d'une part, et les deux Narbonnaises, la Novempopulanie et les Alpes Maritimes, d'autre part, qui répondent à l'inégal développement urbain de ces provinces et à leur histoire différente. Rapportés à l'évangélisation des Gaules, enfin, ces monuments sont relativement tardifs, puisqu'ils relèvent surtout du $V^{e}$ s. et des siècles suivants. Les groupes épiscopaux, qui étaient quasi exclusivement des monuments urbains; les autres édifices de culte urbains, qui relevaient d'une initiative épiscopale ou de l'évergétisme privé; les monastères intra ou extra-urbains; les églises funéraires extra-muros, enfin, sont autant de témoignages de la volonté de l'Église antique d'apposer sa marque sur la topographie urbaine, mais ils valent également par leur apport à la morphogenèse de la ville médiévale et moderne.
\end{abstract}

Key-words. Bishopric, funerary and urban churches, monastery.

Abstract. In the South, in one or two towns out of five the bishopric was during the Antiquity the only Christian monument, and even in the best equipped towns the situation remains slim. Outside the episcopal groups, there are in average only two to four buildings in each town, in which around two thirds were suburban and for $20 \%$ of them included monasteries. In addition, strong regional disparities are found between the two Aquitania and Viennensis in one hand and the two Narbonensis, Novempopulania and Alpes Maritimae in the other hand, corresponding to an uneven urban development of these provinces and to their different history. And finally, these monuments are quite late, inside the frame of the evangelisation of Gaul, since they are mainly dated to the $5^{\text {th }} c$. and following centuries.

The bishoprics, urban monuments for the most part; the other urban cult buildings initiated by bishops or private evergetism; urban or suburban monasteries; and suburban funerary churches at last, are testimonies of the early Christian Church will to print its mark on the urban topography but they altogether are a contribution to the morphogenesis of the medieval and modern town.

Translation: Isabelle FAUDUET

Schlagwörter. Kirchenfamilie, Stadkirche, Grabkirche, Kloster.

Zusammenfassung. Eine oder zwei von fünf südfranzösischen Städten besaßen in der Antike als einziges öffentliches christliches Bauwerk eine Kirchenfamilie und auch für die reicher ausgestatteten Städte fällt die Bilanz bescheiden aus. Außer diesen Baugruppen zählt man nämlich durchschnittlich nur zwei bis vier kirchliche Gebäude pro Stadt. Davon lagen zwei Drittel extra muros, $20 \%$ entfielen auf die Klöster. Dazu kommen große regionale Unterschiede zwischen den beiden Aquitaniae und der Viennensis auf der einen Seite und den beiden Narbonenses, der Novempopulania und den Alpes Maritimae auf der anderen, die mit der ungleichen städtischen Entwicklung in diesen Provinzen und ihrer verschiedenen Geschichte zusammenhängen. Vor dem Hintergrund der Christianisierung Galliens schließlich sind diese Monumente relativ jungen Datums, denn sie gehören vor allem ins 5. Jahrhundert und die darauffolgenden Jahrhunderte.

Die Kirchenfamilien als ausschließlich städtische Großbauten, die übrigen städtischen Kultbauten, die auf bischöfliche Bautätigkeit oder den Euergetismus von Privatleuten zurückgehen, die Klöster intra und extra muros, die Grabkirchen extra muros, all diese Bauwerke sind Zeugnisse für den entschiedenen Willen der antiken Kirche, der Stadttopographie ihren Stempel aufzudrücken, aber sie sind gleichermaßen von Bedeutung für die Gestaltwerdung der mittelalterlichen und neuzeitlichen Stadt.

Übersetzung: Stefan WIRTH 
L'émergence, puis l'affirmation d'une topographie chrétienne, dans les villes d'abord, dans les campagnes ensuite, constitue un des phénomènes les plus caractéristiques de l'Antiquité tardive. H.-I. Marrou, l'un de ceux qui ont le plus efficacement contribué à la diffusion dans notre pays du concept même d' "Antiquité tardive" (Marrou, 1977), l'avait déjà relevé, mais c'est à deux de ses disciples, P.-A. Février et C. Pietri, ainsi qu'à N. Duval, « marrouaque » plus distant, que revient le mérite d'avoir su mettre la chose en évidence. Rassemblant autour d'eux une équipe internationale, ils ont pris pour cela l'initiative d'une enquête intitulée «Topographie chrétienne des cités de la Gaule » qui a d'abord donné lieu à l'élaboration, en 1975 et 1980, de deux recueils de notices provisoires, puis à la parution régulière, depuis 1986, de fascicules successivement édités par N. Gauthier et J.-C. Picard, puis N. Gauthier, B. Beaujard et F. Prévot, dans lesquels sont présentées, en prenant pour cadre les provinces romaines ensuite devenues provinces ecclésiastiques, les villes épiscopales et les monuments chrétiens qui ont contribué à en remodeler le paysage (TCCG, II-XIII, 1986-2004).

Péchant par enthousiasme (ou par souci de stimuler les énergies ?), les promoteurs du projet pensaient qu'il pourrait être mené à bien en une dizaine d'années; en fait, il aura mobilisé les efforts de toute une génération de chercheurs puisque, trente ans après la parution du premier recueil provisoire, deux fascicules restent encore à achever, dont la publication est annoncée comme imminente. Depuis 2004, l'enquête est pourtant terminée pour la Gaule méridionale, ce qui permet de prendre appui sur elle pour jeter sur le phénomène un premier regard avant d'en décliner les modalités par grandes catégories d'édifices construits à l'usage des communautés chrétiennes : groupes épiscopaux, lieux de culte urbains, monastères, basiliques funéraires enfin.

\section{REGARD D'ENSEMBLE SUR LE DIOCĖSE DES SEPT PROVINCES}

À chaque cité son évêque, qui résidait habituellement au chef-lieu : telle était la règle de l'Église antique. La carte administrative des Gaules était cependant mouvante, comme le montre, dans ce dossier de Gallia, la contribution de B. Beaujard (voir supra, p. 11-23) ; retenir pour objet d'étude les villes épiscopales suppose donc de prendre en compte l'ensemble des agglomérations qui furent, à un moment ou un autre de l'Antiquité tardive, des chefslieux de cités, tout en ajoutant à ce corpus d'autres villes, comme Uzès ou Toulon, dont le pragmatisme de la mission chrétienne avait fait des sièges épiscopaux bien qu'elles ne fussent pas des chefs-lieux. Au total, 74 villes sont ainsi à considérer; en témoigne le tableau I sur lequel sont décomptés les monuments chrétiens que ces villes ont, sûrement ou proba-blement, accueillis avant le milieu du VIII $^{\mathrm{e}} \mathrm{s}$.

Avant de tirer les enseignements de ce tableau, dans lequel les données des fascicules II et III (1986), VI et VII (1989), X (1998) et XIII (2004) de la Topographie chrétienne des cités de la Gaule (TCCG) ont naturellement fait l'objet d'indispensables aggiornamenti, quelques remarques sont nécessaires pour une bonne compréhension de la documentation qu'il rassemble. La première colonne, sur les groupes épiscopaux, aurait pu être intégralement renseignée puisqu'il n'est pas douteux qu'à un moment ou à un autre, chaque ville a bien accueilli un tel groupe; on s'est donc borné à noter quelques singularités, comme l'existence dans trois villes de deux groupes épiscopaux, successifs ou concurrents, et à indiquer une datation lorsqu'elle est connue par les textes ou par l'archéologie. Les deux colonnes suivantes rassemblent les édifices classés intra et extra muros par la TCCG, sans que l'on sache trop en réalité si une telle distinction est vraiment opératoire pour les villes de l'Antiquité tardive; la chose ne vaut pas pour la rubrique "monastères", qui réunit les établissements urbains et ceux de la périphérie (à l'exclusion de ceux attachés aux basiliques funéraires). S'agissant enfin des deux colonnes suivantes, la première rassemble les monuments sûrement attestés avant 750, la seconde ceux dont l'interprétation comme édifices chrétiens est problé-matique et ceux qui sont connus par des documents carolingiens ou médiévaux, mais que l'on peut supposer plus anciens.

Ces édifices seulement "possibles» sont au nombre de 101, contre 159 pour les édifices avérés; à les prendre en considération, l'importance à donner à la topographie chrétienne de l'Antiquité tardive s'accroîtrait donc de près des deux tiers. C'est dire quelle est la marge d'incertitude, d'autant qu'il n'est pas sûr que le nombre des monuments qui ont réellement existé soit à inscrire au sein de l'intervalle ouvert entre ces deux estimations, « haute » et «basse »; il peut avoir été plus important, car notre documentation est certainement lacunaire, tributaire qu'elle est du hasard. Hasard des fouilles, cela va sans dire, mais aussi hasard des textes, qui tient à l'inégal intérêt que les auteurs antiques ont porté selon les lieux aux monuments chrétiens et à la conservation, ou non, de leurs écrits : pour ne pas parler d'un Sidoine Apollinaire, d'un Grégoire de Tours ou d'un 
Venance Fortunat, à qui nous devons tant d'indications, songer que la perte de la seule Vita de l'évêque Didier de Cahors amputerait de la moitié le nombre des édifices de cette ville. Malgré ce, quelques traits ressortent avec assez de clarté pour qu'ils ne puissent être mis en doute.

Le premier de ces traits est que l'émergence d'une topographie chrétienne a tenu à quelques édifices choisis. Hors les groupes épiscopaux, le nombre moyen d'établissements dans une ville du Midi est en effet de 3,51 si l'on prend en compte les édifices assurés et ceux dont l'existence est seulement possible, et il tomberait à 2,14 si l'on ne retenait que les monuments sûrement comptabilisables avant 750 . Et le phénomène est loin d'avoir été universel : à ne considérer que les établissements avérés, plus de deux villes sur cinq - 43,24\% exactement - n'auraient compté comme monument chrétien que l'ecclesia, mais il est vrai que la proportion devra être réduite de près de moitié, tombant à $22,97 \%$ si l'on prend également en compte les édifices de chronologie ou d'identification incertaines.

On relèvera d'autre part l'inégale représentation des différentes catégories de monuments que nous avons distinguées. La plus grande part revient aux édifices extraurbains, c'est-à-dire aux églises funéraires et aux sanctuaires des saints : que l'on retienne ou non les monuments attestés après 750, ils représentent les deux tiers des établissements chrétiens (les proportions respectives sont en effet de $66,15 \%$ et $66,66 \%)$. Les monastères, tant urbains qu'extraurbains, fournissent pour leur part environ un établissement sur cinq $(21,38 \%$ à ne considérer que les monuments avérés et $18,84 \%$ pour l'ensemble du matériel). Et - chose qui ne saurait surprendre, en des temps où l'ecclesia était le lieu habituel de rassemblement du peuple chrétien - les établissements intra muros autres que le groupe épiscopal viennent régulièrement en dernier lieu, à hauteur de $11,94 \%$ ou $15 \%$ selon que l'on exclut ou non les monuments douteux ou peut-être postérieurs à 750 .

Sauf pour cette dernière catégorie d'édifices, les proportions restent pourtant pratiquement inchangées, que l'on choisisse d'être rigoureux - d'autres diraient minimaliste - dans l'établissement du corpus de référence, ou que l'on se montre plus généreux en acceptant les édifices dont l'existence est seulement possible. Elles ont donc toute chance de fournir une image assez fidèle de ce qu'était la topographie chrétienne des villes de l'Antiquité tardive dans le Midi : en règle générale, peu ou pas d'édifices intra muros; présence plus ou moins affirmée selon les lieux des établissements monastiques; investissement décidé, enfin, du suburbium par l'Église.

\section{DE FORTES DISPARITÉS PROVINCIALES}

L'approche resterait trop générale si l'on ne faisait droit à de sensibles disparités géographiques, que suffit à mettre en évidence le nombre moyen d'édifices établis dans les villes ou à leurs abords. Entre la province qui compte les villes les mieux dotées et celle dont les villes sont les plus pauvres en monuments chrétiens, l'écart est en effet de plus de un à six - 6,56 exactement - et il atteindrait 9,1 si l'on prenait également en compte les édifices problématiques, ce qui suggère que, loin de se réduire, cet écart a plutôt eu tendance à s'amplifier avec le temps. En témoigne le tableau II, en forme d'histogrammes établis province par province, l'histogramme de gauche prenant en compte les seuls édifices avérés, tandis que celui de droite inclut également les édifices « possibles".

Le trait majeur à retenir de ce tableau est que, quel que soit le corpus de référence, ce sont les mêmes provinces - les deux Aquitaines et la Viennoise d'une part; les deux Narbonnaises, la Novempopulanie et les Alpes Maritimes d'autre part - qui figurent au-dessus et au-dessous de la moyenne; simplement, l'ordre change quelque peu au sein de ces deux sous-ensembles selon que l'on prend ou non en considération les édifices seulement " possibles". Dans le trio de tête, où l'Aquitaine Première conserve toujours le primat, la seconde place oscille en effet entre Aquitaine Seconde et Viennoise selon les cas, tandis que dans le quatuor final, le changement tient au fait que la Narbonnaise Seconde passe de la dernière à la première place si l'on comptabilise les édifices « possibles », qui sont ici particulièrement nombreux -22, comme le montre le tableau I-, tandis que le classement des trois autres provinces, soit, par ordre décroissant, la Narbonnaise Première, la Novempopulanie et les Alpes Maritimes reste invariable.

Il en va donc de ce tableau comme du précédent: les variations qu'il marque quand l'on change de corpus de référence confirment que nous ne pouvons avoir de la topographie chrétienne qu'une image assez approchée, mais les constantes sont telles que cette image, malgré tout, a quelque chance de refléter une réalité. Et autant vaut si, allant plus avant dans l'analyse, on considère le nombre des villes qui, dans chaque province, comptent un nombre déterminé d'établissements chrétiens : 0, 1, 2, 3, etc., comme sur les histogrammes des tableaux suivants, dont le premier ne prend en compte que les édifices assurés, tandis que le second y ajoute les monuments douteux ou connus seulement après 750 (tabl. III). 
Tabl. I - Les monuments chrétiens des villes épiscopales du diocèse des Sept Provinces.

\begin{tabular}{|c|c|c|c|c|c|c|c|}
\hline Provinces & Groupe épiscopal & Édifice urbain & $\begin{array}{c}\text { Édifice } \\
\text { extra-urbain }\end{array}$ & Monastère & $\begin{array}{c}\text { Total édifices } \\
\text { assurés* }\end{array}$ & $\begin{array}{c}\text { Total édifices } \\
\text { possibles }\end{array}$ & Total général* \\
\hline \multicolumn{8}{|l|}{ Viennoise nord } \\
\hline Vienne & 2 , dont 1 arien? & $1 ?$ & $5+6 ?$ & $7+5 ?$ & 12 & 12 & 24 \\
\hline Genève & vers $380 ?$ & $1+1 ?$ & 4 & $1 ?$ & 5 & 2 & 7 \\
\hline Grenoble & fin IVe s.? & & $1+1 ?$ & & 1 & 1 & 2 \\
\hline Alba & ves. & & & & & & $\mathbf{0}$ \\
\hline Viviers & Ve s. & & 4 & & 4 & & 4 \\
\hline Die & & & $1+1 ?$ & & 1 & 1 & 2 \\
\hline Valence & Ve s.-Vle s. & & $2 ?$ & & & 2 & 2 \\
\hline Total & & $1+2 ?$ & $15+10 ?$ & $7+6 ?$ & 23 & 18 & 41 \\
\hline \multicolumn{8}{|l|}{ Viennoise sud } \\
\hline Arles & 2 successifs & 1 & 4 & 4 & 9 & & 9 \\
\hline Saint-Paul-Trois-Châteaux & & & & & & & $\mathbf{0}$ \\
\hline Vaison & $\mathrm{Ve}^{\mathrm{s}} \mathrm{s}-\mathrm{Vle} \mathrm{e}$. & & $1+1 ?$ & & 1 & 1 & 2 \\
\hline Orange & & 1 & 2 & & 3 & & 3 \\
\hline Carpentras & & & & & & & $\mathbf{0}$ \\
\hline Venasque & & & $4 ?$ & & & 4 & 4 \\
\hline Cavaillon & & & $1 ?$ & & & 1 & 1 \\
\hline Avignon & & $1 ?$ & $1+1 ?$ & & 1 & 2 & 3 \\
\hline Marseille & ve $s$. & & $3+4 ?$ & $3+4 ?$ & 6 & 8 & 14 \\
\hline Total & & $2+1 ?$ & $11+11 ?$ & $7+4 ?$ & 20 & 16 & 36 \\
\hline \multicolumn{8}{|l|}{ Aquitaine Première } \\
\hline Bourges & & 2 & $5+2 ?$ & 6 & 13 & 2 & 15 \\
\hline Clermont & & & 17 & 6 & 23 & & 23 \\
\hline Rodez & & $3 ?$ & 2 & $1 ?$ & 2 & 4 & 6 \\
\hline Albi & & & $3+1 ?$ & & 3 & 1 & 4 \\
\hline Cahors & & 4 & 3 & 3 & 10 & & 10 \\
\hline Limoges & & & $3+2 ?$ & $2 ?$ & 3 & 4 & 7 \\
\hline Javols & & & & & & & $\mathbf{0}$ \\
\hline Mende & & & $1+1 ?$ & 1 & 2 & 1 & 3 \\
\hline Saint-Paulien & & & & & & & $\mathbf{0}$ \\
\hline Le Puy-en-Velay & Carolingien? & & $2 ?$ & & & 2 & 2 \\
\hline Total & & $6+3 ?$ & $34+8 ?$ & $16+3 ?$ & 56 & 14 & 70 \\
\hline \multicolumn{8}{|l|}{ Aquitaine Seconde } \\
\hline Bordeaux & après 350 & 2 & $4+2 ?$ & & 6 & 2 & 8 \\
\hline Agen & & & $2+1 ?$ & & 2 & 1 & 3 \\
\hline Angoulême & & & & 1 & 1 & & 1 \\
\hline Saintes & & 1 & 5 & & 6 & & 6 \\
\hline Poitiers & après 350 & & $5+2 ?$ & 1 & 6 & 2 & 8 \\
\hline Périgueux & & & $1+3 ?$ & & 1 & 3 & 4 \\
\hline Rezé & ? (début Ve s.) & & 1 & & 1 & & 1 \\
\hline Total & & 3 & $18+8 ?$ & 2 & 23 & 8 & 31 \\
\hline
\end{tabular}

*non compris les groupes épiscopaux 


\begin{tabular}{|c|c|c|c|c|c|c|c|}
\hline Provinces & Groupe épiscopal & Édifice urbain & $\begin{array}{c}\text { Édifice } \\
\text { extra-urbain }\end{array}$ & Monastère & $\begin{array}{c}\text { Total édifices } \\
\text { assurés* }\end{array}$ & $\begin{array}{c}\text { Total édifices } \\
\text { possibles }\end{array}$ & Total général* \\
\hline \multicolumn{8}{|l|}{ Novempopulanie } \\
\hline Auch & & & 2 & & 2 & & 2 \\
\hline Eauze & & $1+1 ?$ & & $1 ?$ & 1 & 2 & 3 \\
\hline Dax & & & 1 & & 1 & & 1 \\
\hline Lectoure & & & $1 ?$ & & & 1 & 1 \\
\hline Saint-Bertrand-de-Comminges & & $1+1 ?$ & 1 & & 2 & 1 & 3 \\
\hline Saint-Lizier & & & $1 ?$ & & & 1 & 1 \\
\hline Biganos & & & & & & & $\mathbf{0}$ \\
\hline Lescar & & & & & & & $\mathbf{0}$ \\
\hline Aire-sur-l'Adour & & & $1+1 ?$ & & 1 & 1 & 2 \\
\hline Bazas & & $1 ?$ & $1 ?$ & 1 & 1 & 2 & 3 \\
\hline Tarbes & & & 2 & & 2 & & 2 \\
\hline Saint-Lézer & & & & & & & $\mathbf{0}$ \\
\hline Oloron-Sainte-Marie & & & & & & & $\mathbf{0}$ \\
\hline Total & & $2+3 ?$ & $7+4 ?$ & $1+1 ?$ & 10 & 8 & 18 \\
\hline \multicolumn{8}{|l|}{ Narbonnaise Première } \\
\hline Narbonne & avant 441 & $1+1 ?$ & $4+1 ?$ & & 5 & 2 & 7 \\
\hline Toulouse & & 3 & $2+2 ?$ & & 5 & 2 & 7 \\
\hline Béziers & & & $2 ?$ & & & 2 & 2 \\
\hline Elne & & $1 ?$ & $1 ?$ & & & 2 & 2 \\
\hline Agde & & & 3 & & 3 & & 3 \\
\hline Maguelone & & & 1 & & 1 & & 1 \\
\hline Nîmes & & $2 ?$ & $2+1 ?$ & & 2 & 3 & 5 \\
\hline Lodève & & & & & & & $\mathbf{0}$ \\
\hline Carcassonne & & & $1 ?$ & & & 1 & 1 \\
\hline Uzès & & & $2+1 ?$ & & 2 & 1 & 3 \\
\hline Arisitum & & & & & & & $\mathbf{0}$ \\
\hline Total & & $4+4 ?$ & $14+9 ?$ & & 18 & 13 & 31 \\
\hline \multicolumn{8}{|l|}{ Narbonnaise Seconde } \\
\hline Aix-en-Provence & 2 successifs, le $2^{\mathrm{e}}$ vers 500 & & $1+5 ?$ & & 1 & 5 & 6 \\
\hline Apt & & $1 ?$ & $3 ?$ & & & 4 & 4 \\
\hline Riez & $\mathrm{Ve}_{\mathrm{e}} \mathrm{s}$ & $1+1 ?$ & 2 & & 3 & 1 & 4 \\
\hline Fréjus & $\mathrm{Ve}_{\mathrm{e}}$ & 1? & $1 ?$ & & & 2 & 2 \\
\hline Gap & & & $2 ?$ & & & 2 & 2 \\
\hline Sisteron & & $1 ?$ & $2 ?$ & & & 3 & 3 \\
\hline Antibes & ve s. & & $2 ?$ & & & 2 & 2 \\
\hline Toulon & & $2 ?$ & $1 ?$ & & & 3 & 3 \\
\hline Total & & $1+6 ?$ & $3+16 ?$ & & 4 & 22 & 26 \\
\hline \multicolumn{8}{|l|}{ Alpes Maritimes } \\
\hline Embrun & & & 2 & & 2 & & 2 \\
\hline Digne & fin $V e s$. & & 1 & & 1 & & 1 \\
\hline Nice & & & & & & & 0 \\
\hline Cimiez & ve s. & $1 ?$ & 1 & $1+1 ?$ & 2 & 2 & 4 \\
\hline Vence & & & & & & & 0 \\
\hline Glandèves & & & & & & & 0 \\
\hline Castellane & & & & & & & 0 \\
\hline Thorame & & & & & & & 0 \\
\hline Senez & & & & & & & 0 \\
\hline Total & & $1 ?$ & 4 & $1+1 ?$ & 5 & 2 & 7 \\
\hline Total général & & $19+20 ?$ & $106+66 ?$ & $34+15 ?$ & 159 & 101 & 260 \\
\hline
\end{tabular}


Tabl. II - Nombre moyen d'édifices chrétiens par ville dans les Sept Provinces, à l'exclusion du groupe épiscopal.

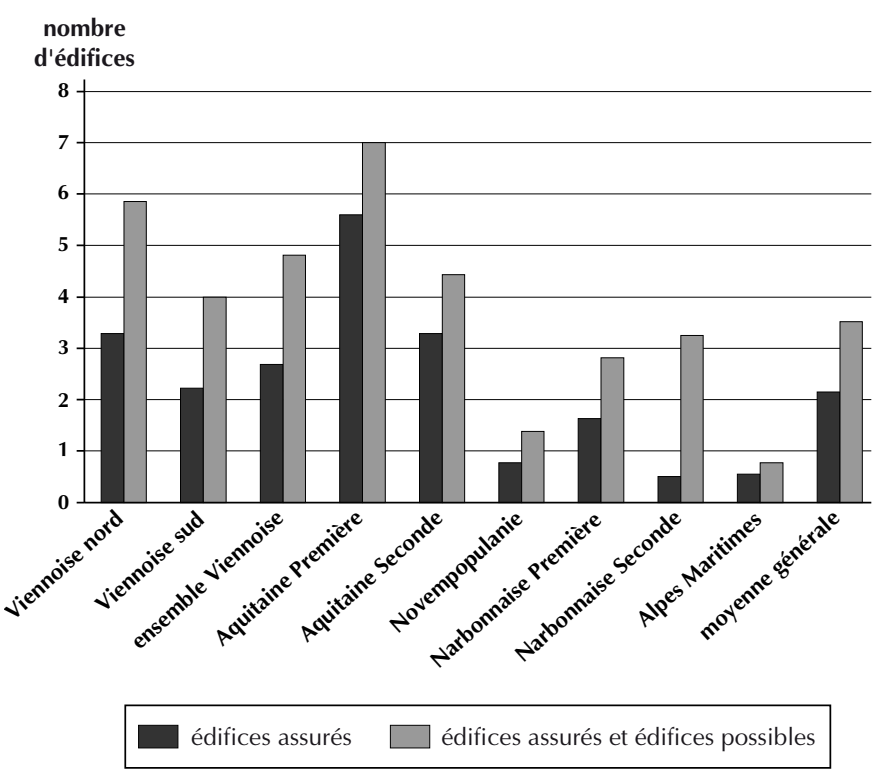

L'exception comme la règle ressortent de l'examen conjoint de ces deux tableaux. L'exception tient aux villes qui apparaissent isolées à droite, au nombre de quatre ou cinq, et il est à noter que, même si l'ordre varie quelque peu, il s'agit à chaque fois, ou presque, des mêmes villes : Clermont, Bourges et Vienne, qui figurent en tête dans les deux tableaux, d'une part; Cahors, Arles et Marseille, d'autre part, qui ne se détachent à leurs côtés que dans l'un ou l'autre tableau, mais figurent toujours dans la mouvance du groupe de tête. Sauf pour Cahors, où la documentation, comme je l'ai dit, est largement tributaire des comptages minutieux du testament de l'évêque Didier et de l'auteur de la Vita du saint, cette liste a donc toute chance de rassembler les villes épiscopales majeures de la Gaule méridionale.

Que ces villes soient toutes en Aquitaine Première ou en Viennoise contribue largement au fait que ces deux provinces occupent les premiers rangs dans la série des histogrammes du tableau II, mais ne suffit pas à expliquer cette prééminence, qui tient également à d'autres villes situées en bonne place en partie gauche des tableaux III et IV. Si l'on observe qu'inversement, sur ces mêmes tableaux, les provinces les plus déshéritées du tableau II se recommandent par une égale médiocrité de leurs villes épiscopales, on en déduira comme règle plus générale que les villes d'exception ne constituent que les plus riches fleurons de provinces bien dotées par ailleurs.
Il est loisible, ainsi, d'établir une sorte de palmarès provincial, dans lequel on ne s'étonnera pas, comme on l'a dit, que figurent en tête à la fois la Viennoise et ces deux Aquitaines que Salvien tenait pour la «moelle des Gaules » et, aux dernières places, la Novempopulanie et les Alpes Maritimes, dans lesquelles les Romains n'avaient que partiellement réussi à établir un véritable réseau urbain; comme ce réseau a servi d'ossature à celui des villes épiscopales, la hiérarchie ainsi esquissée répond bien à ce que l'on sait par ailleurs de ces provinces et de leur importance. La surprise vient en revanche du rang relativement médiocre qui revient aux deux Narbonnaises, pourtant anciennement romanisées et anciennement christianisées, et le fait qu'au sein de la Viennoise, la partie méridionale, ou province ecclésiastique d'Arles, participe d'une tendance analogue paraît montrer qu'il ne s'agit pas là d'un biais dû à notre documentation, mais d'un trait qui se retrouve dans l'ensemble du Midi méditerranéen. S'intéresser à une autre variable - le temps -, qui est au moins aussi importante que l'espace, peut fournir un élément d'explication pour ce fait surprenant.

\section{UNE TARDIVE ET PROGRESSIVE CONQUÊTE DE L'ESPACE URBAIN}

Même si, grâce à Vienne, la région compte l'une des premières Églises qui soit connue dans les Gaules, en 177 , le processus de christianisation a été relativement tardif et surtout assez lent en Gaule méridionale. Sans doute l'ensemble des provinces - sauf peut-être la Novempopulanie - comptaient-elles au moins une Église au début $\mathrm{du} \mathrm{IV}^{\mathrm{e}} \mathrm{s}$., comme en témoigne la liste des signatures au concile d'Arles de 314; le réseau des villes épiscopales restait pourtant sans doute encore embryonnaire à la fin du siècle et même s'il a été amplement étoffé au cours du siècle suivant, il n'est pas sûr qu'il ait été achevé partout au tournant des années 500, certains diocèses, dans des régions marginales comme les Alpes par exemple, n'étant sûrement attestés qu'au VI ${ }^{\mathrm{e}} \mathrm{s}$.

Mais l'émergence d'une topographie chrétienne a été plus tardive encore, comme le montre le décalage qui se vérifie fréquemment entre la chronologie fournie dans le tableau I à propos des groupes épiscopaux connus par l'archéologie et la date de première attestation de l'évêché : ainsi en Provence, où la plupart des édifices sont attribués à un " $\mathrm{V}^{\mathrm{e}}$ siècle taillé large ", comme aimait à dire P.-A. Février, alors qu'ils relèvent de sièges certainement plus anciens. La chose surprendra si l'on a en mémoire 
Tabl. III - Les villes épiscopales des Sept Provinces et le nombre des édifices chrétiens qu'elles ont sûrement accueillis avant 750, à l'exclusion du groupe épiscopal.

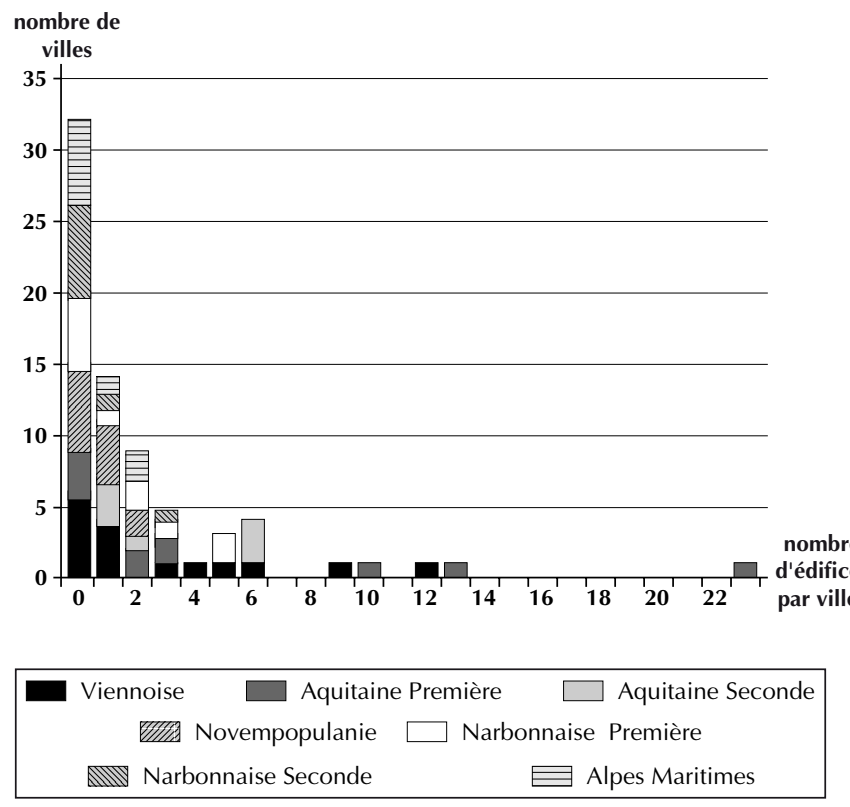

le texte fameux d'Eusèbe de Césarée (Histoire ecclésiastique, VIII, 1, 5) signalant que dès le début du IV ${ }^{\mathrm{e}} \mathrm{s}$., dans chaque ville, "sortaient du sol de belles et vastes églises " en lieu et place des "maisons de prière " d'antan, mais c'est qu'Eusèbe avait l'Orient à l'esprit quand il écrivait cela. En Gaule méridionale, ce n'est pas avant les dernières décennies du $\mathrm{IV}^{\mathrm{e}} \mathrm{s}$., en l'état actuel de nos connaissances, que les communautés chrétiennes ont eu le désir, les moyens financiers et le poids politique suffisants pour élever des cathédrales qui constituaient autant de signes ostensibles de leur foi.

Mais il en est de même, voire davantage, pour l'ensemble des édifices chrétiens, comme en font foi les notices de l'atlas archéologique des Premiers monuments chrétiens de la France (Duval dir., 1995 et 1996), dont les deux premiers volumes fournissent un bilan à peu près exhaustif, arrêté en 1995 pour le Sud-Est et en 1996 pour le Sud-Ouest et le Centre : qu'il s'agisse des cathédrales ou d'autres édifices, ces notices ne comptent guère de monuments antérieurs au $\mathrm{V}^{\mathrm{e}} \mathrm{s}$., qui a inauguré une sorte d'âge d'or pour le premier art chrétien dans le Midi. Car une fois le processus engagé, il n'a plus connu de trêve. Le fait est bien connu pour le $\mathrm{VI}^{\mathrm{e}}$ s., grâce notamment à Grégoire de Tours, mais il vaut sûrement pour les siècles ultérieurs. En témoignent dans le tableau I tous les monuments qui sont réputés comme seulement «possibles »: le fait que l'on hésite à placer
Tabl. IV - Les villes épiscopales des Sept Provinces et le nombre des édifices chrétiens qu'elles sont susceptibles d'avoir accueillis avant 750, à l'exclusion du groupe épiscopal.

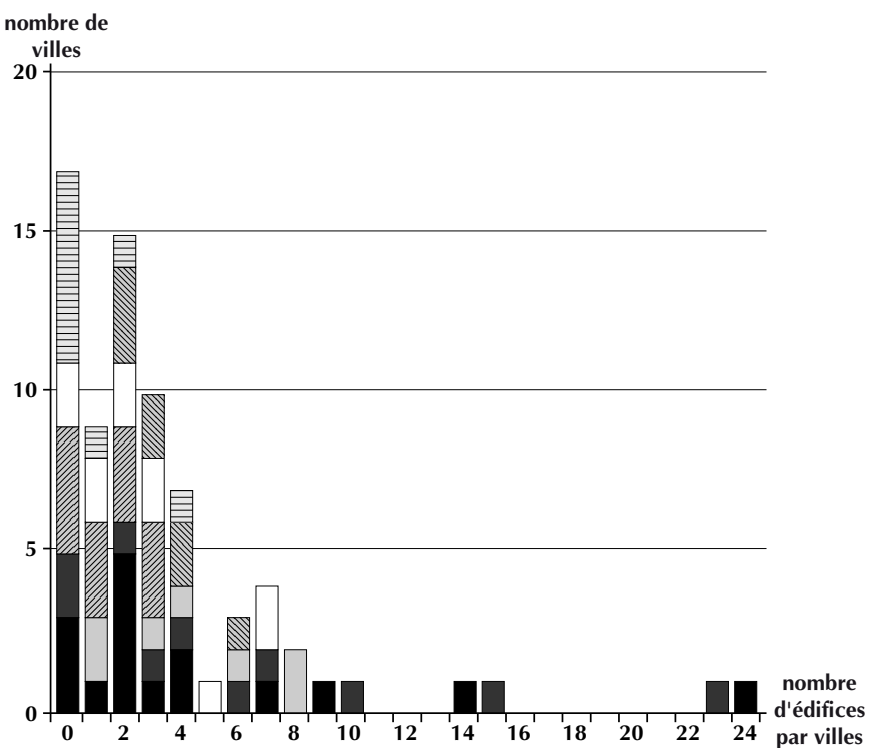

autant d'édifices avant ou après 750 dit assez quelle importance le VIII $^{\mathrm{e}} \mathrm{s}$. lui-même a eue pour l'affirmation d'une topographie chrétienne dans les villes.

Or si l'on remarque que les fondations les plus tardives ont souvent été le fait des princes mérovingiens, de leurs familiers ou de l'aristocratie qui leur était attachée, peutêtre tient-on là un élément d'explication à la surprenante modestie (relative) de la parure monumentale des villes de Viennoise méridionale et des deux Narbonnaises, qui ont rarement accueilli de telles fondations parce qu'elles étaient les plus éloignées des nouveaux centres du pouvoir et, donc, les moins ouvertes à leur influence. Ces cités qui avaient été des lieux privilégiés, comme l'archéologie en témoigne, pour la constitution du premier établissement chrétien n'ont que peu ou pas été touchées par la seconde phase de cet établissement, qui annonçait la « renaissance carolingienne » hors de notre sujet.

En voilà assez pour ce survol topochronologique de la documentation; il reste maintenant à revenir aux rubriques du tableau I afin d'examiner les différents types de monuments constitutifs de la topographie chrétienne et de brosser à grands traits la problématique qui ressort des recherches récentes à leur sujet. 


\section{LES GROUPES ÉPISCOPAUX}

Une précision pour commencer : «groupe épiscopal» est une appellation moderne, et même très récente, qui sert à désigner l'ensemble des monuments indispensables à la vie d'une communauté chrétienne, à savoir : les édifices de culte dans lesquels les fidèles se rassemblaient habituellement autour de leur évêque, cathédrale et baptistère, d'une part; la résidence de l'évêque (et de ses clercs s'ils vivaient avec lui en communauté), mais aussi des locaux de service, voire d'assistance, d'autre part. Tous ces éléments étaient désignés dans l'Antiquité par les termes d'ecclesia (parfois basilica) et baptisterium pour les premiers, domus ecclesiae ou domus ecclesiastica pour les seconds, mais quel que soit le vocabulaire que l'on privilégie, il renvoie à des réalités qui étaient sûrement assez diverses selon les lieux.

\section{DES CONFIGURATIONS DIVERSES}

Si le terme de " groupe » a été mis en circulation par les archéologues pour désigner les bâtiments de l'episcopium, c'est parce que ces bâtiments étaient fréquemment réunis en un même lieu. Pour preuve, les nombreux témoignages issus de la documentation littéraire ou de données de fouille qui forment la matière des notices de la TCCG auxquelles, pour ne pas multiplier les références, on ne fera pas de renvoi explicite. Pour l'archéologie, qu'il suffise de songer au cas de Genève, sur lequel on trouvera dans ce dossier de Gallia un éclairage de C. Bonnet (voir infra, p. 111-115), et pour les textes à celui de Clermont, où la résidence épiscopale était si proche de la cathédrale que l'évêque Gallus pouvait, sur son lit de mort, entendre les chants de l'office, mais il en allait de même à Arles, Cahors et bien d'autres villes.

Il est pourtant des documents qui permettent d'entrevoir des dispositions plus « éclatées ", comme à Marseille où Grégoire de Tours signale au $\mathrm{VI}^{\mathrm{e}}$ s. l'existence de plusieurs domus ecclesiae, sans que l'on puisse savoir, il est vrai, si cette pluralité répondait à un dispositif originel ou à un accroissement du pouvoir temporel de l'Église locale avec les années. Or les conséquences sur la topographie urbaine sont tout autres selon que le groupe épiscopal constituait un ensemble compact ou qu'il répondait à des éléments plus ou moins disséminés dans le tissu urbain; qu'il suffise pour le mesurer de rapprocher le plan du groupe épiscopal de Genève de celui d'Aix-en-Provence (fig. 42).

Dans le premier cas, c'est tout le quadrant nord-est de la ville qui a été remodelé pour faire place aux établissements chrétiens; à Aix-en-Provence en revanche, le réseau des rues a subsisté dans ses grandes lignes et si une insula tout entière, à l'ouest, a été radicalement modifiée du fait de l'installation de la cathédrale et de ses annexes en lieu et place de monuments publics à l'abandon ou désaffectés pour l'occasion, il n'est pas sûr que l'aspect du pâté de maison situé plus à l'est, de l'autre côté d'un cardo secondaire, ait été sensiblement affecté quand l'évêque - du moins peut-on le supposer - réaménagea dans le même temps une domus pour en faire sa résidence. Le hasard des fouilles veut que la Gaule méridionale offre ainsi deux images, sinon opposées, du moins sensiblement différentes, de ce que pouvait être un groupe épiscopal, mais on serait bien en peine de multiplier les exemples, Genève et Aix-en-Provence constituant, au sein de la liste du tableau I, les deux ensembles de loin les mieux connus par l'archéologie.

Cette liste est d'ailleurs des plus courtes : une vingtaine d'édifices à peine, parfois réduits à des restes presque insignifiants. C'est dire que dans trois villes sur quatre, on ignore encore tout des groupes épiscopaux antiques - et souvent jusqu'à leur existence même, les sources antérieures à 750 ne mentionnant qu'assez rarement l'ecclesia ou ses annexes. S'agissant d'éléments aussi indispensables aux Églises et à leur mission, nul doute pourtant que ces groupes ont bien existé et même que l'on puisse imaginer de les localiser en règle générale à l'emplacement des cathédrales actuelles. Cela tient à l'expérience tirée des fouilles faites sous ces édifices, qui livrent le plus souvent des résultats positifs, mais aussi à un argument de bon sens. À quelque époque que ce fût, il a fallu en effet de sérieuses raisons pour déplacer un groupe épiscopal; la meilleure preuve en est que dans le Midi, au cours de l'Antiquité tardive, de tels déplacements se comptent au nombre de deux, soit 2,7\% seulement de notre corpus.

La chose est assurée pour Arles où la cathédrale fut transférée, sans doute au V ${ }^{\mathrm{e}} \mathrm{s}$., de l'angle sud-est du rempart vers le site de l'actuelle primatiale Saint-Trophime (fig. 50 ; voir à ce sujet Heijmans, infra, p. 121-124). Elle est seulement probable pour Aix-en-Provence où les fouilles qui ont été faites sous l'actuelle cathédrale Saint-Sauveur ont permis de dater du tournant des années 500 les premiers monuments chrétiens du site, ce qui invite à chercher ailleurs un premier groupe épiscopal. Comment imaginer en effet que cette ville, qui avait été promue au rang de métropole dans le dernier quart du $\mathrm{IV}^{\mathrm{e}}$ s., soit restée sans cathédrale jusqu'à une date aussi basse ? À cause de sa titulature, l'église médiévale de Notre-Dame-de-la-Seds paraît toute désignée pour avoir été le siège de cette ecclesia primitive et 

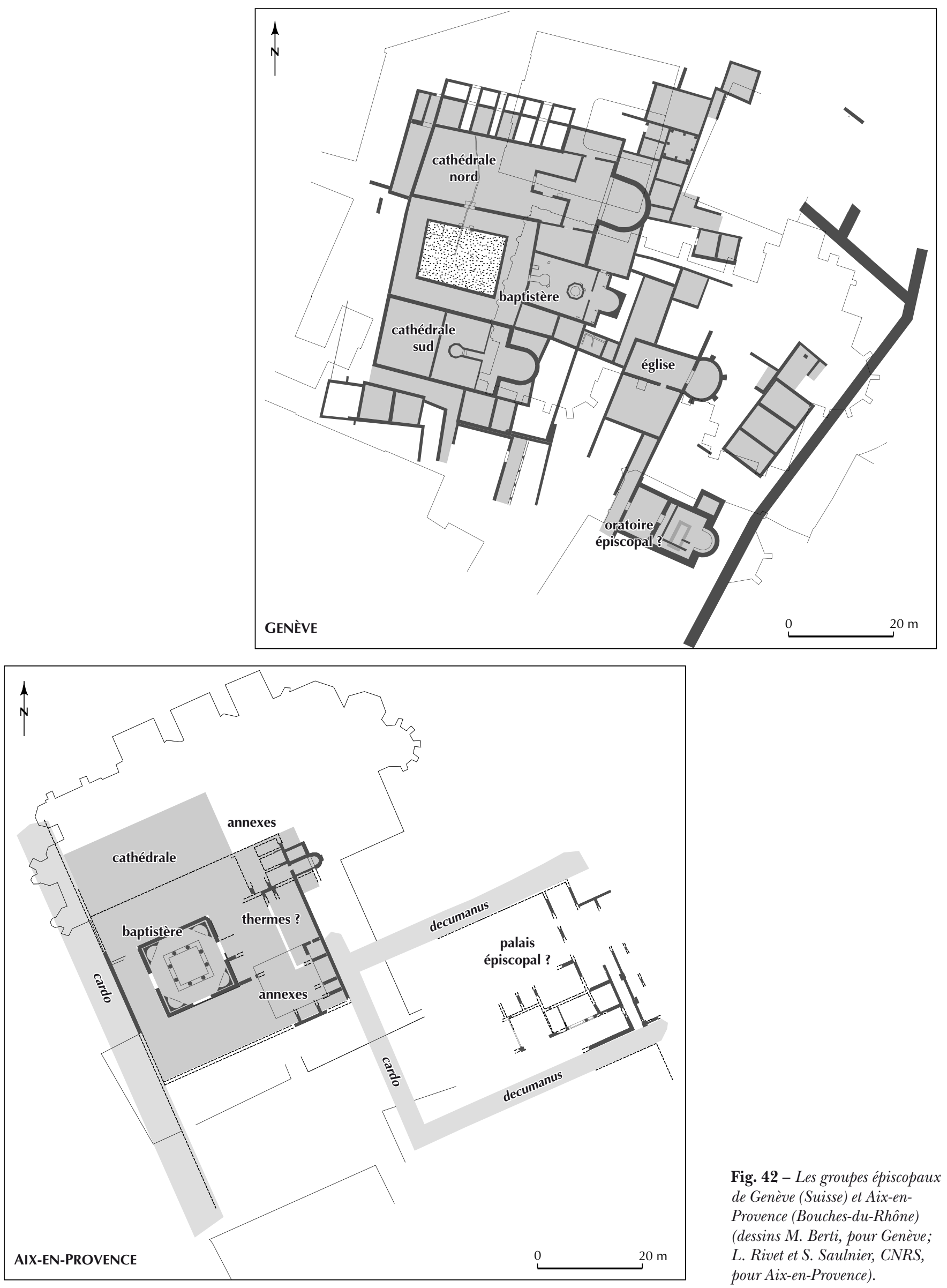

Fig. 42 - Les groupes épiscopaux de Genève (Suisse) et Aix-enProvence (Bouches-du-Rhône) (dessins M. Berti, pour Genève; L. Rivet et S. Saulnier, CNRS, pour Aix-en-Provence). 


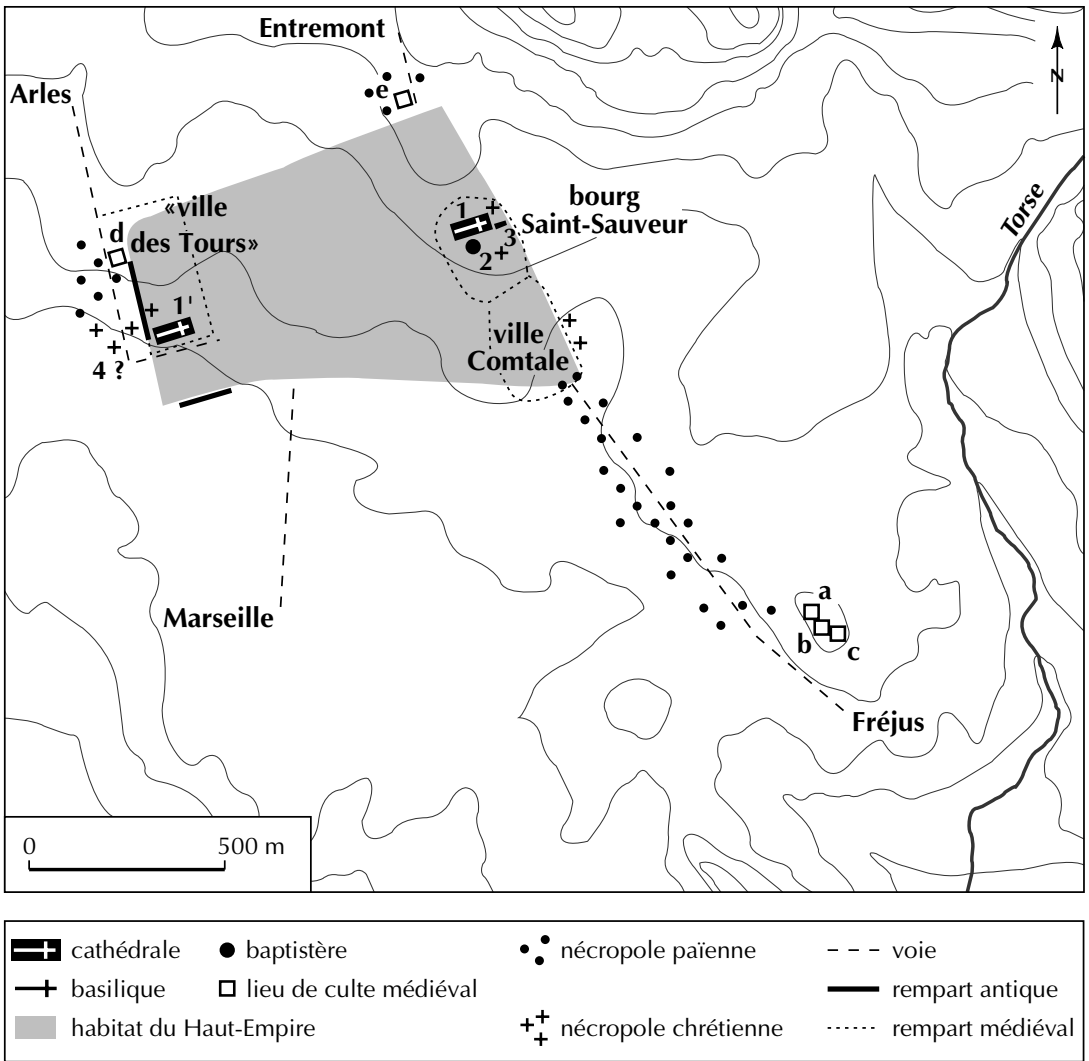

Fig. 43 - Aix-en-Provence (Bouches-du-Rhône). Topographie chrétienne pendant l'Antiquité tardive : en pointillés, les contours, certains ou probables, des bourgs médiévaux (les numéros renvoient à la liste des édifices chrétiens de la TCCG, II, 1986a, p. 19) (dessin S. Roucole). Édifices antiques : 1', cathédrale primitive (Notre-Dame-de-la-Seds ?); 1, cathédrale du VI ${ }^{e}$ s. (Saint-Sauveur) ; 2, baptistère ; 3, résidence épiscopale? ; 4, tombe de saint Mitre ? Édifices de date incertaine : a-c, Saint-Pierre, Saint-Sauveur du Puy, Saint-Étienne; $d$, Saint-Laurent ; e, Saint-André. l'hypothèse est d'autant plus vraisemblable qu'à la retenir, le transfert opéré à Aix-en-Provence serait analogue à celui qui est avéré pour Arles. Dans les deux cas en effet, il aurait conduit au déplacement vers le centre de la ville d'un episcopium initialement installé à la périphérie de l'agglomération (fig. 43). À la périphérie, mais intra muros, et ce qui vaut pour Arles et pour Aix-en-Provence vaut pour les autres groupes épiscopaux du Midi, qui étaient eux aussi des édifices proprement urbains.

\section{DES ÉTABLISSEMENTS URBAINS}

Que les groupes épiscopaux soient à considérer décidément comme des édifices urbains constitue l'un des acquis majeurs à porter à l'actif de l'enquête sur la « Topographie chrétienne des cités de la Gaule ». Elle a fait justice en effet d'une historiographie encore très en vogue jusque dans les années 1970, qui imaginait en règle générale extra muros les premières cathédrales. Une telle reconstruction se fondait sur quelques indications ambiguës glanées dans des textes antiques et surtout sur les faux diplomatiques forgés au Moyen Âge par les abbayes suburbaines soucieuses d'asseoir leurs prétentions face aux évêques et à l'église épiscopale en prétendant avoir été le siège primitif de l'ecclesia locale. Mais elle ne résiste pas à l'analyse : qu'il suffise de se reporter aux notices des différents fascicules de la TCCG, et en particulier à celle que $\mathrm{F}$. Prévot a rédigée à propos du groupe épiscopal de Clermont, qui a longtemps passé pour une sorte de cas d'école d'établissement extra muros.

Si l'on passe sur des cas où l'aire remparée (mais non forcément agglomérée) est difficile à établir, comme à Béziers, l'unique exception avérée, dans le Midi, pourrait être constituée par Dax, où un texte des années 1052-1057 atteste d'un transfert de l'ecclesia de l'église Saint-Vincentde-Xaintes, située aux abords de la ville, vers "l'intérieur des murs de la cité »- c'est-à-dire de l'enceinte antique encore conservée aujourd'hui. Pour autant, si rien n'autorise à douter d'un tel témoignage, rien ne dit non plus que la situation ainsi décrite pour le milieu du $\mathrm{XI}^{\mathrm{e}} \mathrm{s}$. ait été identique à celle de l'Antiquité tardive. Comment s'étonner, d'ailleurs, qu'en règle générale, les groupes épiscopaux aient été établis au sein même des villes, quand ils avaient pour fonction de rassembler la communauté des fidèles et de pourvoir à sa desserte spirituelle ? De ce fait, leur insertion 
dans le tissu urbain n'est pas allée sans difficulté, comme le montrent les constructions qu'il a fallu araser pour leur faire place et dont les fouilles ont quelquefois gardé trace. À Poitiers, ainsi, dans la seconde moitié du IV ${ }^{\mathrm{e}}$ s., il s'agit de bâtiments dévolus à l'habitat et à l'artisanat et, dans les dernières décennies du siècle, à Genève, d'une résidence aristocratique ou officielle; $\mathrm{au} \mathrm{V}^{\mathrm{e}} \mathrm{s}$., à Cimiez ou à Riez, ce sont des bâtiments publics - des thermes en l'occurrence; à Aix-en-Provence enfin, au tournant des années 500, un temple (ou plus probablement une basilique civile) et la place qui lui était liée. La gradation que marque cette succession chronologique entre appropriation de demeures privées, puis de bâtiments publics, et, pour finir, conquête de l'espace public est-elle le fruit du hasard? Elle traduit bien en tout cas que si l'Église a acquis au fil du temps une absolue maîtrise sur la topographie urbaine, cette acquisition n'a pourtant été que progressive.

Cela explique que rares sont les groupes épiscopaux, tels ceux nouvellement construits à Arles au $\mathrm{V}^{\mathrm{e}} \mathrm{s}$. et à Aix-en-Provence au tournant des années 500, qui ont été établis à proximité ou au détriment d'une partie du centre monumental. Là où la configuration des villes est à peu près connue - ce qui est loin d'être toujours le cas -, ces premiers établissements chrétiens occupent en effet le plus souvent une position marginale, dans un angle du rempart (comme à Bordeaux ou à Arles pour le groupe épiscopal primitif), tout près de la courtine (comme pour le premier groupe aixois, ceux de Bourges, Clermont, Angoulême, Grenoble, Toulouse et tant d'autres) ou sont situés en limite de la zone agglomérée dans des villes ouvertes (comme Cimiez, Limoges ou Riez) ; les rares cas où l'on relève une position un peu plus centrale tiennent à des villes telles que Vienne, Orange, Cahors, Poitiers, Nîmes, Apt ou Fréjus. Ces diverses localisations auraient pu être résumées par un tableau mais l'exercice serait sans grande signification, les villes de l'Antiquité tardive étant souvent si exiguës qu'un édifice, où qu'il soit situé, n'est jamais très éloigné à la fois du rempart... et du centre de l'agglomération.

Mais même dans les villes les plus importantes, et si marginaux qu'ils fussent parfois, les groupes épiscopaux ne constituaient pas moins des signes fort ostensibles de la foi chrétienne. Songer par exemple au premier groupe épiscopal d'Arles, qui était sûrement le monument le plus apparent pour les voyageurs découvrant la cité depuis la route de Marseille parce que l'angle de l'enceinte dans lequel il avait été cantonné coïncide, ou peu s'en faut, avec le sommet de la butte sur laquelle la ville est établie; comme sur ces représentations de villes - vignettes de documents officiels ou panneaux de mosaïque que donne à voir, pour d'autres régions que les Gaules, l'art de l'Antiquité tardive -, sa silhouette se détachait ainsi très au-dessus des crénelages de la courtine. Or un même souci d'ostentation se retrouvait probablement dans la plupart des autres villes, ce qui explique le soin que les Anciens ont apporté à l'architecture des groupes épiscopaux.

\section{DES PROGRAMMES ARCHITECTURAUX COMPLEXES}

Faisons retour sur le groupe épiscopal de Genève (fig. 42) qui constitue une référence obligée, et pas seulement pour la Gaule méridionale, grâce à l'étendue et à la qualité des fouilles dont il a fait l'objet. Laissant de côté la résidence épiscopale, pour laquelle on ne pourrait invoquer dans le Midi que les possibles (et apparemment plus modestes) parallèles fournis par Aix-en-Provence ou Fréjus, on s'attachera aux seuls édifices de culte; cela est amplement suffisant pour évoquer la complexité des programmes architecturaux mis en œuvre pour l'érection d'un groupe épiscopal. Cette complexité provient d'abord du nombre des édifices : l'ensemble comptait sûrement ici deux cathédrales (dont l'une, au nord, date des dernières décennies $\mathrm{du} \mathrm{IV}^{\mathrm{e}} \mathrm{s}$., tandis que l'autre, au sud, est un peu plus tardive) entre lesquelles ont pris place un et même - chose exceptionnelle - deux baptistères; à quoi l'on adjoindra, au sud-est, un autre édifice à abside outrepassée et, dans la mouvance du palais épiscopal, un probable oratoire de l'évêque. Mais la complexité tient également à d'incessantes reconstructions (voir Bonnet, infra, fig. 55) ; pour autant, même si l'édifice nord s'est trouvé privilégié au terme de tous ces travaux, on trouve bien dans les multiples monuments que compte le site la traduction architecturale des titulatures, également complexes, que nous font connaître pour les groupes épiscopaux les documents écrits d'époque carolingienne ou médiévale.

Ces titulatures avaient suggéré à J. Hubert l'idée que nombre de cathédrales antiques des Gaules étaient des " cathédrales doubles» (Hubert, 1963) et cette thèse, comme l'illustration qu'en donne le groupe épiscopal de Genève, ont souvent servi, implicitement ou explicitement, à imaginer ce que pouvaient être les autres groupes épiscopaux du Midi qui n'offrent pas, à beaucoup près, des éléments aussi bien attestés : voir les notices des Premiers monuments chrétiens de la France, pour Grenoble par exemple, voire Lyon, de peu situé hors des limites des Sept Provinces, auxquelles on adjoindra, afin de ne pas en rester 
à l'arc alpin, les reconstructions prudemment avancées par Q. Cazes pour le groupe épiscopal de Toulouse (Cazes, 1998, p. 20-23). Et les découvertes les plus récentes invitent assez à aller dans la même voie, comme en témoignent dans ce dossier les contributions de W. Migeon sur les fouilles de Bordeaux (voir infra, p. 117-119) et de M. Heijmans sur celles de l'enclos Saint-Césaire à Arles (voir infra, p. 121-124) : les absides monumentales que ces fouilles ont livrées sont plus que des pièces à ajouter au puzzle des groupes épiscopaux de ces deux villes majeures; elles apportent la preuve qu'ils comptaient sûrement, eux aussi, de nombreux édifices de culte.

Pour autant, on ne saurait faire de l'existence de « cathédrales doubles » une règle générale du premier art chrétien : une table ronde qui a fourni la matière d'un dossier de la revue Antiquité tardive l'a opportunément rappelé voici dix ans (Duval, Caillet dir., 1996). S'agissant du Midi, que l'on en juge par l'exemple de la Provence où certaines cathédrales, comme celles de Riez ou de Cimiez, ne comptaient apparemment qu'un vaisseau et où la liste des édifices doubles dont P.-A. Février avait cru pouvoir supposer l'existence sous des cathédrales romanes qui comptent deux nefs juxtaposées doit sans doute être réévaluée à la baisse (Février, 1964, p. 55-57). Cela vaut au moins pour Fréjus, où l'une des deux nefs est au mieux une construction du haut Moyen Âge, tandis que dans les autres monuments qui ont été fouillés récemment, à Antibes et Aix-en-Provence, les reconnaissances sont restées trop incomplètes pour que la restitution d'un édifice double originel ne puisse dépasser le stade d'hypothèse. C'est dire que l'on ne saurait songer à une typologie uniforme pour les cathédrales, et pas davantage imaginer partout la reproduction de programmes plus ou moins analogues à celui que nous fait connaître Genève; cela est si vrai qu'au moins autant que la complexité, c'est la diversité qui constitue la principale caractéristique des groupes épiscopaux antiques.

\section{UNE DIVERSITÉ RECHERCHÉE}

Pour donner la mesure de la diversité des groupes épiscopaux, on se tournera vers le bâtiment qui en était sans doute l'élément le plus symbolique: le baptistère, dans lequel une fois l'an en principe, pendant la nuit de Pâques, était administré le sacrement de l'initiation chrétienne, qui était gage de la croissance et de la pérennité des Églises. Une étude récente sur l'ensemble des baptistères gaulois a mis en lumière que, parmi ceux qui étaient suffisamment connus par l'archéologie, il n'en était pas deux qui fussent exactement semblables (Guyon, $2000)$; la chose vaut particulièrement pour le Midi, qui offre en la matière un observatoire privilégié, puisqu'il compte tous les baptistères de France encore conservés en élévation, et la toute récente découverte du baptistère de Limoges, sur laquelle on lira d'autre part dans ce dossier une contribution de J. Denis (voir infra, p. 125-129), ne peut que confirmer ce jugement.

Cette diversité vaut pour les piscines creusées dans le sol qui étaient au cœur de ces monuments le plus souvent de plan centré, qu'il s'agisse de leur profondeur, de leur taille ou de leur configuration, même si l'on note une nette préférence pour les tracés octogonaux, dont la large diffusion s'explique peut-être par des raisons symboliques, le chiffre huit étant celui de la résurrection promise aux fidèles qui avaient été baptisés, comme le dit l'apôtre Paul : «dans la mort et la résurrection du Christ ».

Mais la diversité tient également au plan des édifices, qui ont d'ailleurs souvent été aussi fréquemment transformés que les cathédrales : les fouilles de Genève en témoignent, mais l'on pourrait également invoquer le cas de Poitiers où des recherches récentes, encore en cours, restituent sans cesse plus précisément une évolution complexe (BoissavitCamus, 2004) et plus encore celui de Grenoble, dont la découverte constitue l'un des acquis majeurs des dernières décennies (Baucheron et al., 1998, p. 81-104). Les plans successifs adoptés par le baptistère de cette ville offrent en effet une sorte de raccourci des principaux schémas attestés par ailleurs, depuis la simple salle rectangulaire des origines jusqu'à l'édifice à la fois cruciforme et polylobé du dernier état, en passant par ce vaisseau augmenté à l'est d'une abside qui est attesté dès le deuxième état; tout cela pour ne pas parler des transformations plus ou moins simultanées de la piscine, qui sont au moins aussi complexes (fig. 44).

Également variable enfin était la place qu'occupaient au sein des groupes épiscopaux ces bâtiments qui devaient être isolés ou facilement isolables afin de préserver le caractère secret du baptême : qu'ils aient été accolés à l'édifice de culte ou séparés de lui par un espace ouvert, ils pouvaient être à l'ouest de la cathédrale, comme à Riez, Poitiers ou Grenoble; au sud, comme à Aix-en-Provence; au nord, comme à Cimiez ou Marseille; entre les deux vaisseaux d'une cathédrale double, enfin, comme à Genève et peutêtre Toulouse. On le voit, toutes les solutions possibles ont été mises en œuvre.

Il ne faudrait pas exagérer cependant ces dissemblances, car la variété des formes disponibles pour les piscines et les 


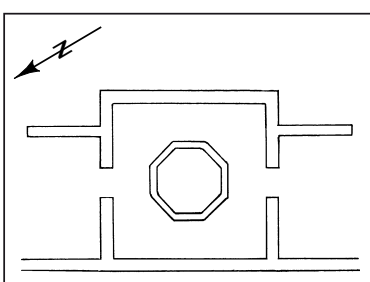

état 1 du baptistère phase 1 de la cuve

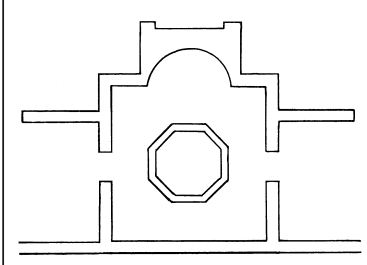

état 2 du baptistère phase 1 de la cuve

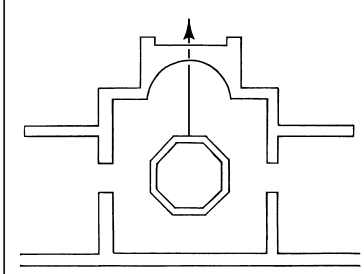

état 2 du baptistère phase 2 de la cuve

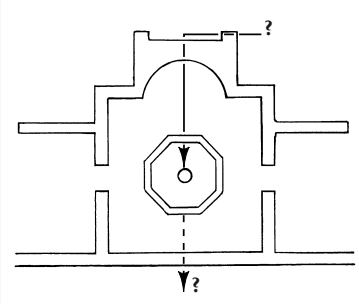

état 2 du baptistère phase 3 de la cuve

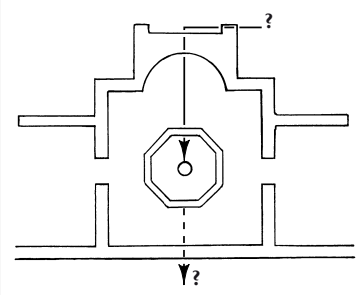

état 2 du baptistère phase 4 de la cuve

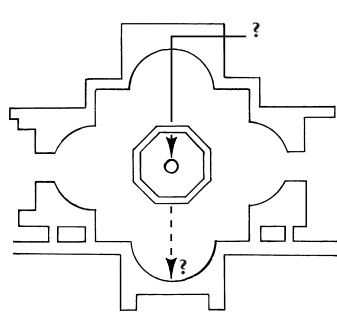

état 3 du baptistère phase 4 de la cuve

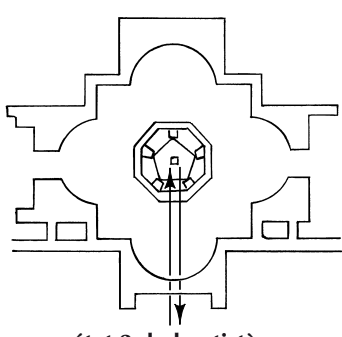

état 3 du baptistère phase 5 de la cuve

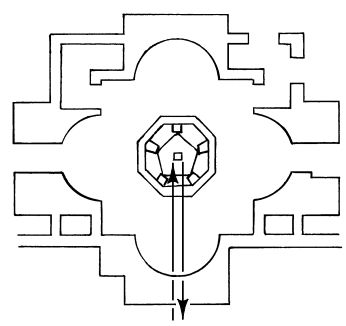

état 4 du baptistère phase 5 de la cuve

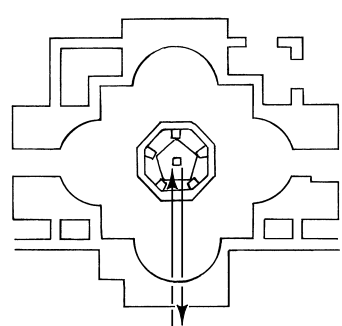

état 5 du baptistère phase 5 de la cuve

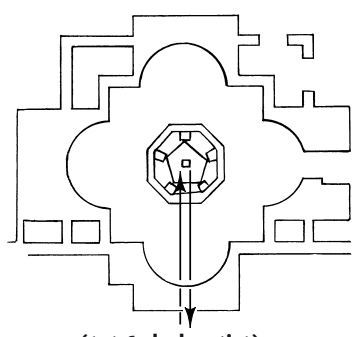

état 6 du baptistère phase 5 de la cuve salles baptismales n'était pas infinie, de sorte que s'il est vrai qu'il n'est pas dans le corpus des baptistères aujourd'hui connus dans le Midi deux bâtiments identiques, les différences tiennent souvent à peu de choses. La meilleure preuve en est donnée par la série des baptistères de Provence, qui doivent à leur bon état de conservation (sauf pour Marseille) d'avoir de longue date été étudiés et reconnus comme des sortes de types architecturaux (Guyon, 1989b). De fait, leurs architectes paraissent s'être livrés à de subtiles variations autour d'un thème unique, celui d'une salle carrée à l'extérieur, octogonale à l'intérieur et pourvue (ou non) d'un déambulatoire annulaire, mais l'important est surtout de noter les dimensions très diverses de leurs constructions : une centaine de mètres carrés de superficie hors œuvre pour Cimiez, Riez et Fréjus; plus du double pour Aix-en-Provence et trois fois plus encore à Marseille, dont la salle de plus de $600 \mathrm{~m}^{2}$ n'a d'équivalent qu'hors des frontières des Gaules, à Milan par exemple (fig. 45).

Ces différences d'échelle seraient encore plus marquées si l'on prenait également en compte les élévations, qui étaient évidemment à proportion; il est douteux qu'elles reflètent exactement la (probable) inégale importance des communautés chrétiennes locales, mais elles reproduisent fidèlement en revanche une hiérarchie ecclésiastique implicite ou explicite: les trois premières villes nommées étaient en effet de simples villes épiscopales, tandis qu'Aix-enProvence était métropole; Marseille, enfin, bien qu'elle fût une simple cité de Viennoise, comptait une Église qui ambitionnait au tournant des années 400 de régenter l'épiscopat de Narbonnaise Seconde et, plus largement, de toute la Provence orientale.

Il apparaît donc séduisant de voir dans la taille donnée à ces édifices une façon pour leurs Églises d'affirmer leur rang ou d'afficher leurs prétentions et les découvertes récemment faites à Arles, cette grande rivale de Marseille dans la région, vont bien dans le sens d'une telle hypothèse. Sans doute l'abside que les fouilles d'Arles ont livrée ne relève-t-elle pas d'un baptistère, mais possiblement de la première cathédrale de la ville; pour autant, les dimensions de cette abside sont aussi hors normes que peuvent l'être celles du baptistère de Marseille, les parallèles étant à nouveau à chercher hors des Gaules, à Rome ou à Milan. Prolongeant ou reprenant à son compte cette émulation entre cités qui fut l'un des

Fig. 44 - Grenoble (Isère). Évolution du baptistère (dessin F. Pont, INRAP, d'après Baucheron et al., 1998, fig. 73, p. 103). 

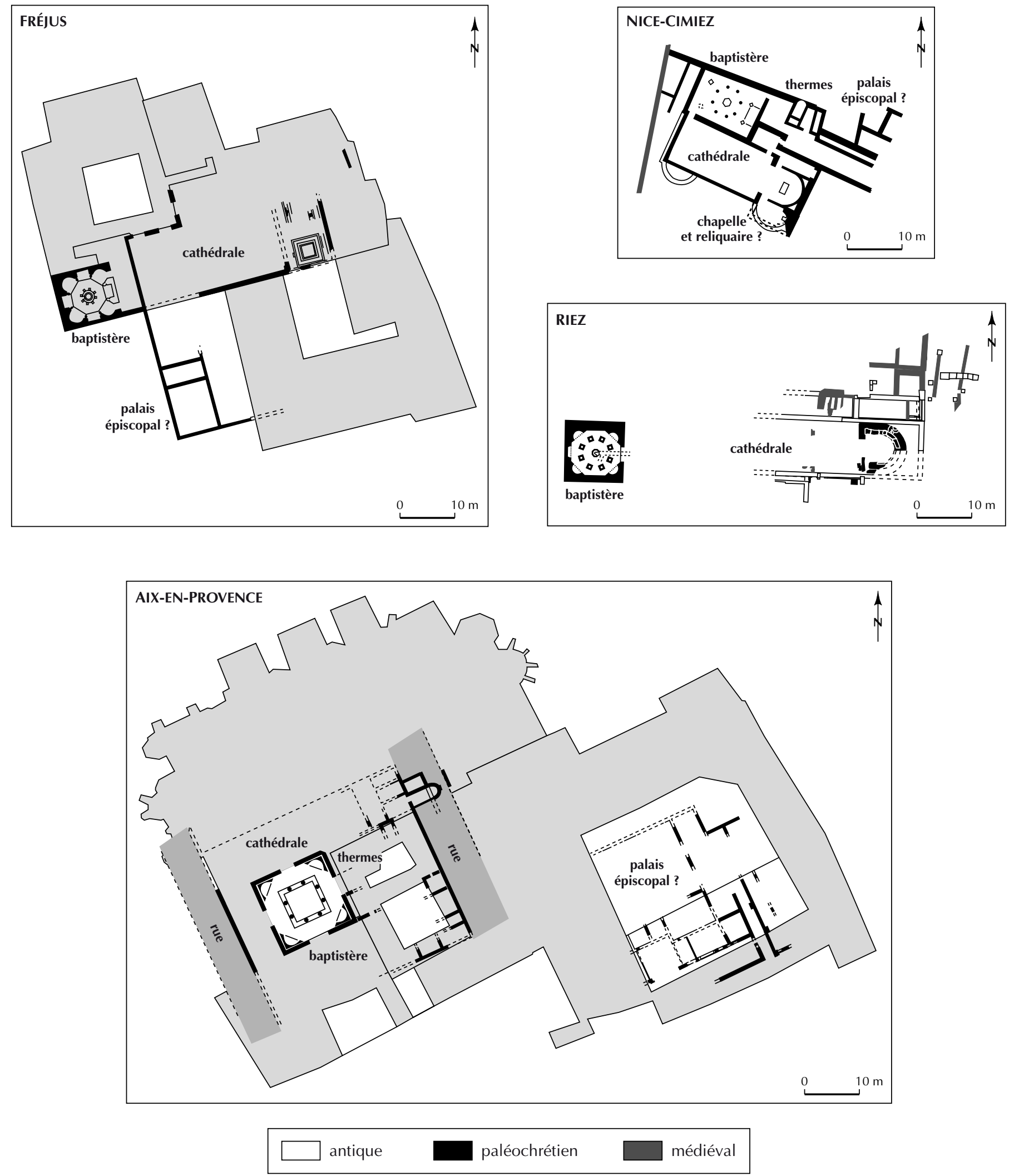
plus puissants ressorts du monde antique, la concurrence entre Églises était donc apparemment des plus vives, et l'on comprend sans peine qu'elle ait trouvé dans les groupes épiscopaux un élément de choix pour s'exercer. Pourtant, dans la majorité des villes, comme on l'a dit, ces groupes ne constituaient pas le seul élément d'une topographie chrétienne.

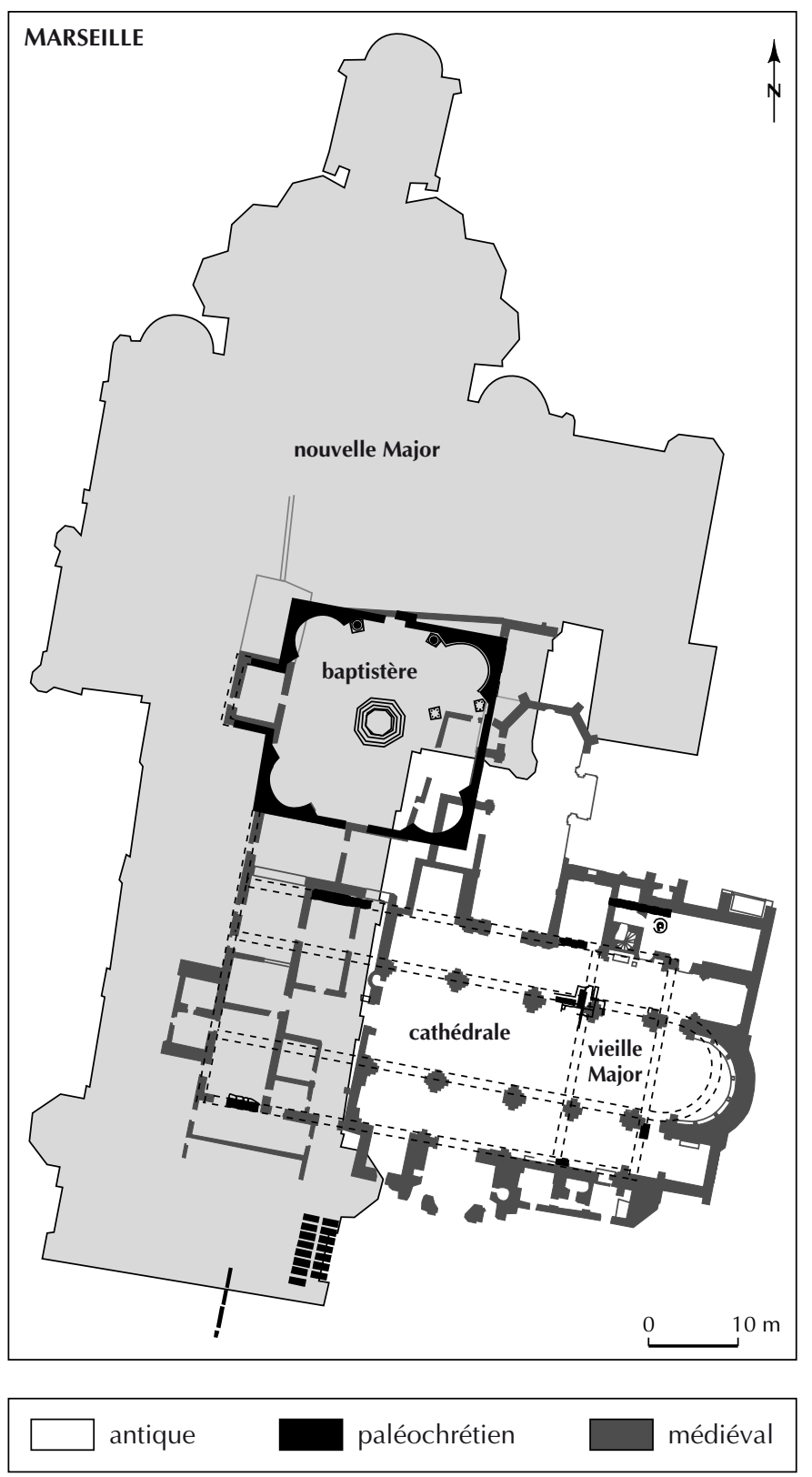

Fig. 45 - Les groupes épiscopaux de Fréjus (Var), Cimiez (Alpes-Maritimes), Riez (Alpes-de-Haute-Provence), Aix-en-Provence (Bouches-du-Rhône) et Marseille (Bouches-du-Rhône) (dessin C. Hussy, SRA PACA, d'après Delestre dir., 2005, p. 191-192).

\section{LES AUTRES ÉDIFICES DE CULTE URBAINS}

Sur le tableau I, la colonne où sont recensés les édifices urbains n'est pas seulement la moins renseignée, elle est également celle qui compte la plus forte proportion d'édifices «possibles », au point que le nombre de ces édifices dépasse, fût-ce de peu, celui des monuments qui sont sûrement attestés avant 750. S'il est donc bien vrai que l'investissement de l'espace intra muros par la topographie chrétienne est ce phénomène assez discret que l'on a dit, le nombre élevé d'édifices de culte que l'on hésite à situer précisément dans le cours du VIII ${ }^{\mathrm{e}} \mathrm{s}$. montre pour sa part que cet investissement s'est sûrement accru avec le temps. Comment s'en étonner? Il participe largement de la deuxième phase de l'établissement chrétien dans les Gaules, qui annonce déjà les temps médiévaux, au cours desquels des édifices de culte autres que les cathédrales sont devenus des éléments obligés du paysage urbain. Les contemporains étaient d'ailleurs peut-être déjà sensibles à cet état de fait car la mention ecclesiae (au pluriel) se retrouve à deux reprises chez Grégoire de Tours quand il brosse, pour Bazas et Saint-Bertrand-de-Comminges, des «portraits de villes" encloses par leur rempart; il est vrai pourtant que la formulation est ambiguë : s'applique-t-elle aux différents édifices de culte de ces cités, comme je serais enclin à le penser, ou aux composantes de leur groupe épiscopal, groupe que Grégoire désigne pourtant d'ordinaire par le terme ecclesia, au singulier ? On l'ignore.

L'impact de ces édifices urbains sur la topographie a pu être des plus modestes quand il s'agissait d'oratoires que des particuliers avaient installés au sein de leur maison, comme cela s'est rencontré à Bordeaux ou à Saintes, voire de maisons transformées en basiliques, comme à Bourges. Il n'est pas sûr en effet que le décor urbain ait été affecté, si peu que ce soit, par ces installations ou ces transformations et il en allait sans doute de même pour certaines créations ex novo, comme cet oratoire Saint-Martin que l'évêque Didier avait fait construire à Cahors, car la modestie du terme employé pour désigner l'édifice laisse à penser qu'il s'inscrivait probablement de façon discrète dans le paysage. Il est impossible en revanche de deviner l'importance de la plupart des autres édifices urbains, que les sources désignent généralement comme des basiliques: ce terme, comme on sait, pouvait recouvrir les réalités les plus diverses et l'archéologie n'aide guère à l'éclairer, car rares sont les édifices urbains qui sont connus par une fouille. 
En ce domaine, Genève offre pourtant une fois encore un exemple privilégié grâce à la basilique Saint-Germain, qui était assez éloignée du groupe épiscopal pour qu'elle ait marqué par sa présence, dès le début $\mathrm{du} \mathrm{V}^{\mathrm{e}} \mathrm{s}$., un autre quartier de la ville (voir Bonnet, infra, p. 114); à quoi il faudrait peut-être ajouter ici une autre basilique connue par un sermon prononcé par Avit de Vienne lors de sa dédicace, au début du $\mathrm{VI}^{\mathrm{e}}$ s., mais que l'on ne sait où situer. Ce bilan peut paraître limité; en fait, il égale, dépasse même parfois, celui des cinq villes que nous avons qualifiées de « majeures » au sein des Sept Provinces. Notre documentation n'indique en effet aucun édifice urbain autre que l'episcopium pour Marseille et Clermont, un seul pour Arles et Vienne, et si deux maisons remployées comme basiliques permettent à Bourges d'égaler Genève, il s'agissait, on l'a dit, d'établissements probablement modestes et d'ailleurs tardifs, puisqu'ils relèvent des dernières décennies du VII ${ }^{\mathrm{e}} \mathrm{s}$. Qu'en déduire, sinon que l'investissement des villes par une topographie chrétienne a sans doute été pour beaucoup un phénomène contingent?

Les indications que les sources écrites fournissent à l'occasion sur les fondations urbaines vont assez dans le sens de cette interprétation. La construction des groupes épiscopaux, du moins peut-on le supposer, a dû être pour l'essentiel le fait des évêques, qui n'hésitaient pas cependant, pour ce faire, à solliciter la générosité des autorités et de l'aristocratie convertie: l'inscription que Rusticus de Narbonne avait fait graver sur le linteau de sa cathédrale afin de célébrer, le 29 novembre 445, la reconstruction de l'ecclesia après un incendie fournit un exemple classique de ces évergésies. Il en a été un peu différemment pour les autres églises urbaines : beaucoup, sans doute, étaient également des fondations épiscopales, comme ces quatre basiliques qu'énumère le testament de Didier de Cahors ou encore la basilique anonyme de Genève, que l'intitulé de l'homélie d'Avit de Vienne permet d'attribuer à l'évêque Maximus, mais également nombreuses sont celles qui relevaient d'autres initiatives, privées ou publiques.

À la première catégorie se rattachent les oratoires déjà cités de Bordeaux et de Saintes, et les maisons qui furent transformées en basiliques à Bourges par la volonté d'une certaine Eustadiola. La seconde catégorie est plus fournie encore; elle compte des constructions ex novo, comme la basilique construite par le patrice et préfet Libère à Orange en 529 et peut-être la basilica Constantia d'Arles, sûrement antérieure d'un (bon ?) siècle, mais que M. Heijmans juge être une basilique civile qui n'aurait été que momentanément transformée en lieu de culte (Heijmans, 2004a, p. 263). Pour des dates plus tardives, on relèvera également des édifices de culte installés dans des temples plus ou moins transformés pour l'occasion, ce qui requérait l'autorisation de la puissance publique : la chose paraît assurée à Toulouse pour la basilique Saint-Pierre-Saint-Géraud que le duc Launebaude avait élevée à l'emplacement du Capitole (voir supra, fig. 6, p. 29) afin de conserver la mémoire de l'arrestation de Saturnin, le premier évêque de la ville, mort martyr sous Dèce (Arramond et al., 1993) ; elle est possible à Vienne pour le temple d'Auguste et Livie, qui doit peutêtre à sa transformation en édifice de culte pendant l'Antiquité tardive ou le haut Moyen Âge d'être encore conservé aujourd'hui. Et comment ne pas mentionner enfin l'église Notre-Dame-de-la Daurade, à Toulouse, que l'on interprétera volontiers comme la chapelle attachée au probable palais que les fouilles de l'hôpital Larrey ont permis d'identifier en 1988 (voir supra, fig. 16, p. 41 ; cf. Pailler dir., 2001, p. 489-490)?

Une question encore ouverte est de savoir quelle était la fonction de ces divers édifices. La réponse paraît évidente pour ceux qui relevaient de fondations privées et pour la possible chapelle palatine de Toulouse : ils étaient prioritairement destinés aux dévotions de leurs commanditaires et de leurs familiers. Quant aux sanctuaires comme celui qu'avait fondé Launebaude à Toulouse, ils étaient sans doute plus largement ouverts aux fidèles, lors des fêtes des saints et des martyrs, au même titre que ceux, beaucoup plus nombreux, qui étaient installés dans les cimetières de la périphérie comme on le dira bientôt, mais qu'en était-il des autres ? Les recherches récentes sur la basilique du quartier du Plan à Saint-Bertrand-de-Comminges apportent peutêtre un élément de réponse.

La chose pourra surprendre quand cet édifice est surtout connu, depuis sa découverte au début du siècle dernier, pour les sarcophages qu'il abritait. La reprise des fouilles suggère pourtant de voir en lui une église proprement urbaine, qui n'a acquis sa fonction funéraire qu'au terme d'une évolution plus complexe que ne l'imaginaient ses découvreurs (fig. 46). Il a en effet été élevé au sein d'une insula encore densément habitée dans les premières décennies $d \mathrm{~V} \mathrm{~V}^{\mathrm{e}} \mathrm{s}$., sans doute à peu près au même moment que le groupe épiscopal, et ses dimensions - près d'une trentaine de mètres de long à l'origine, $45 \mathrm{~m}$ à l'issue de ses transformations - sont dignes d'une église épiscopale : il serait donc séduisant de voir en lui la basilique de la ville basse, qui répondait dans la plaine à la cathédrale installée sur la hauteur (fig. 47). L'hypothèse est d'autant plus plausible qu'une configuration similaire se vérifie dans 

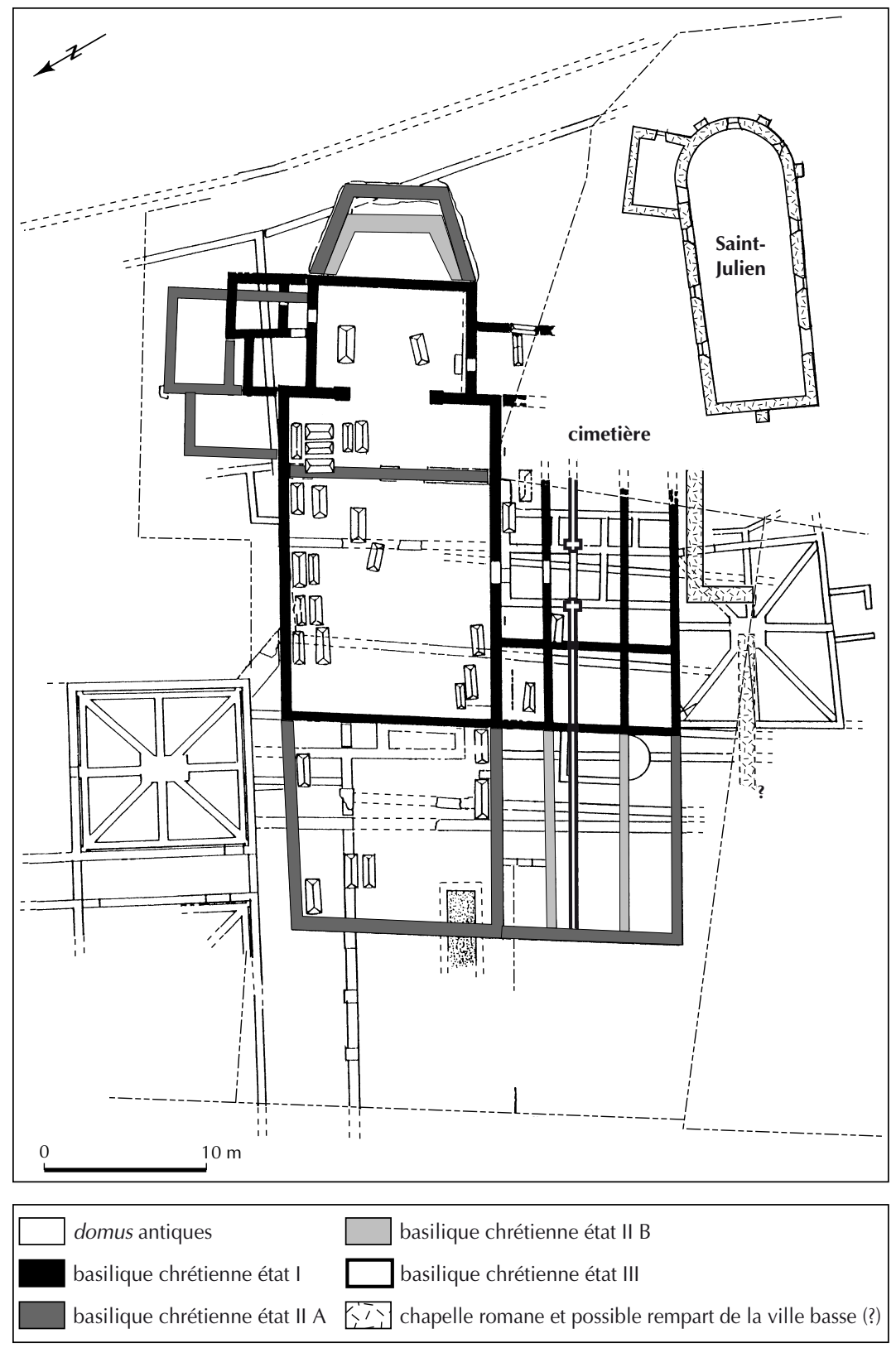

Fig. 46 - Saint-Bertrand-de-Comminges (Haute-Garonne). La basilique chrétienne et ses annexes à l'issue des fouilles des années 1985-1991 (dessin J.-L. Paillet, CNRS, d'après TCCG, XIII, 2004, p. 75).

d'autres villes qui allient pareillement un site de piémont à un site perché : ainsi, peut-être, à Saint-Lizier tout proche, où les « concathédrales » médiévales de Notre-Dame-de-laSède, sur la butte, et de Saint-Lizier en contrebas ont pu succéder à des établissements plus anciens; ainsi aussi, plus sûrement, à Riez où le même schéma se vérifie, mais à fronts renversés, l'église Saint-Albin, sur la hauteur, répondant ici à la cathédrale située dans la plaine. Or le cas de Riez est d'autant plus intéressant que le biographe de l'évêque Maxime dépeint les pérégrinations pieuses que son héros, au milieu du $\mathrm{V}^{\mathrm{e}}$ s., accomplissait dans les basiliques de sa ville, y compris Saint-Albin, qui est précisément mentionnée. Faut-il voir là, sinon l'attestation, du moins l'anticipation de cette liturgie stationale de l'Église ancienne, que l'on connaît bien pour la Rome de l'Antiquité tardive, mais dont on ne discerne dans les Gaules que des linéaments 


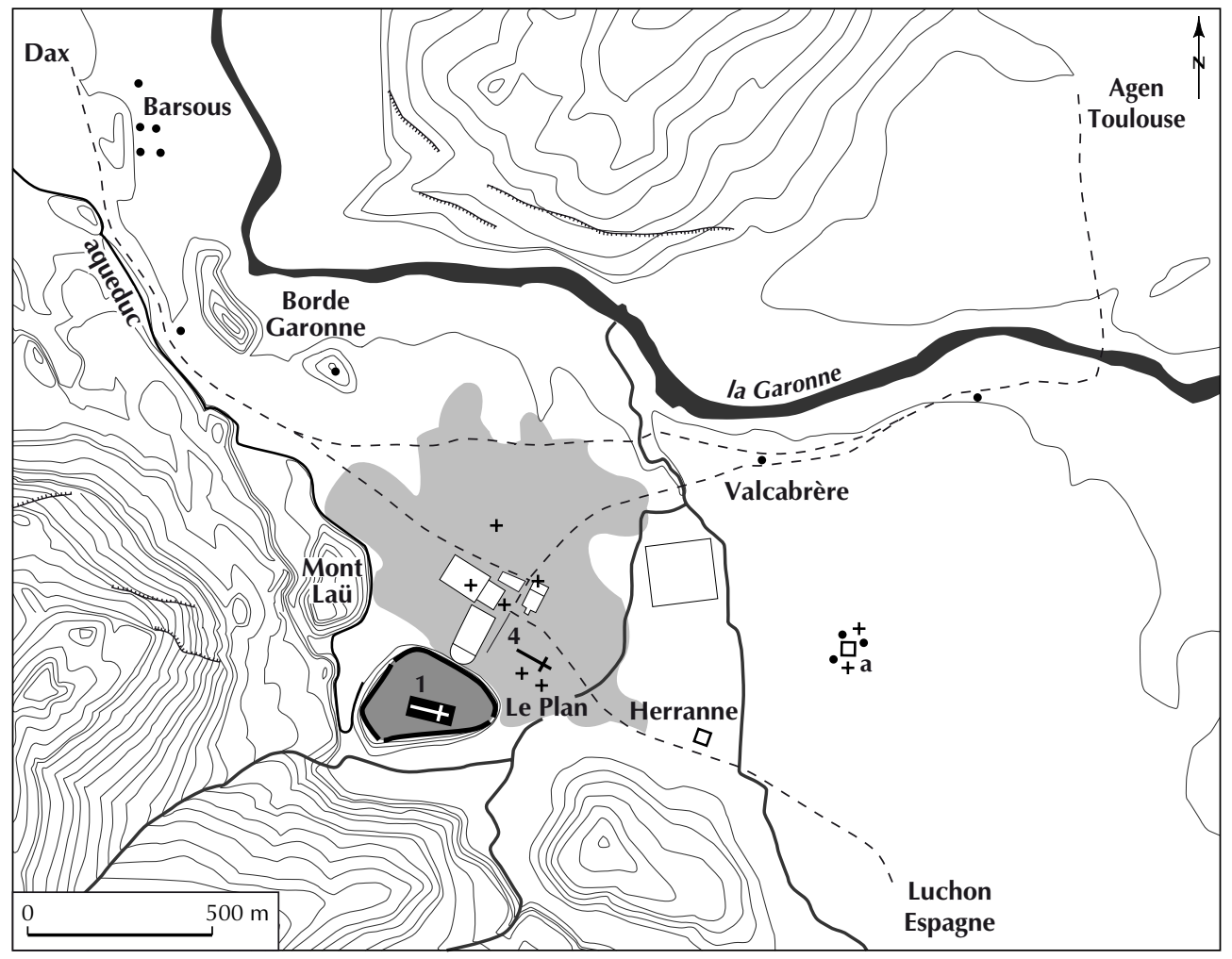

\begin{tabular}{|c|c|c|c|}
\hline \# cathédrale & - baptistère & $\because$ nécropole païenne & --- voie \\
\hline + basilique & $\square$ lieu de culte médiéval & & \\
\hline habitat du Haut-Empire & habitat de la fin de l'Antiquité & $+_{+}^{+}$nécropole chrétienne & _ rempart antique \\
\hline
\end{tabular}

Fig. 47 - Saint-Bertrand-de-Comminges (Haute-Garonne). Topographie chrétienne pendant l'Antiquité tardive (les numéros renvoient à la liste des édifices chrétiens de la TCCG, XIII, 2004, p. 53) (dessin V. Gémonet, CNRS). Édifices antiques : 1, cathédrale; 4, basilique du quartier du Plan. Édifice de date incertaine : a, basilique funéraire de Saint-Just.

bien plus tardifs (Saint-Roch, 1989) ? Cela serait sans doute aventureux, mais ce témoignage montre au moins quel était le souci des évêques de l'Antiquité tardive d'étendre leur pastorale à l'ensemble des églises de la ville où ils résidaient. Ainsi s'ébauchait le processus qui devait conduire, au terme de toute une évolution, à la création des paroisses urbaines au Moyen Âge.

\section{LES MONASTÈRES}

Autant la date d'apparition des premiers groupes épiscopaux et des premiers édifices de culte urbains reste assez problématique, autant celle des premiers monastères est précisément connue. Un peu plus tardive que dans la Gaule du Nord, où Martin s'était illustré par ses initiatives pionnières, elle a coïncidé dans le Midi avec les premières décennies du $V^{e}$ s., qui furent marquées d'abord par l'installation d'Honorat à Lérins, qui est hors de notre propos, puis par celle de Jean Cassien à Marseille, où il fonda deux monastères, l'un d'hommes et l'autre de femmes, que l'on ne sait trop où situer, faute de savoir quel crédit accorder à des traditions médiévales qui font du second un établissement urbain et du premier une fondation extra-urbaine installée à Saint-Victor, face à la ville, sur l'autre rive du Lacydon.

Même si certains ont supposé que l'évêque Proculus avait peut-être sollicité la venue de Cassien, ces fondations ne relevaient donc pas d'une initiative proprement épiscopale, et ce trait s'est maintenu tout au long de la période. Que l'on songe à cette Eustadiola dont nous avons déjà mentionné combien ses évergésies avaient doté Bourges à la fin $\mathrm{du} \mathrm{VII}^{\mathrm{e}} \mathrm{s}$. : aux deux basiliques qu'elle avait aménagées, 
il faut ajouter maintenant un monastère de femmes dont elle assura d'ailleurs la direction. L'une des particularités du monachisme est cependant que même s'il était né d'une volonté de rupture avec le monde, il a très vite conquis l'establishment: les carrières de Martin en Gaule du Nord ou d'Honorat dans le Midi sont exemplaires à cet égard, puisque tous deux ont terminé leur vie comme évêques - et évêques de villes aussi importantes que Tours et Arles. Or, devenus évêques, les moines ont souvent fondé dans leurs villes des monastères, inaugurant en cela une tradition qui ne s'est pas interrompue elle non plus; elle s'est même renforcée avec le temps, quand la prière continue des communautés monastiques a été recherchée comme un secours efficace pour le repos des morts. De là ce que l'on pourrait appeler un second établissement monastique, qui tient à des fondations installées auprès des basiliques funéraires (ou créées avec ces basiliques), aussi bien par les évêques que par des notables soucieux de leur salut, ce qui fait qu'en définitive les monastères relèvent $d$ 'initiatives aussi diverses que les églises urbaines.

Ce second établissement monastique n'a été recensé dans le tableau I ni dans la colonne relative aux monastères ni dans celle des édifices extra-urbains, où ont été décomptées pour une seule unité les basiliques auxquelles était attachés un abbé ou une communauté; la raison en est que dans une étude à visée topographique, multiplier les occurrences n'aurait pas eu grand sens, s'agissant de fondations établies en un même lieu ou en des lieux très proches. Un inconvénient pourtant à cela : la part qui revient au monachisme dans l'investissement du monde urbain par l'Église s'en trouve réduite, et singulièrement pour les périodes les plus tardives. En témoigne le nombre relativement réduit des bâtiments monastiques réputés seulement «possibles » sur ce tableau; il aurait été sensiblement accru si l'on avait également pris en compte les établissements liés aux basiliques car nombreux sont ceux qui ne sont connus que par des sources carolingiennes ou plus tardives.

Le nombre des villes qui ont compté des monastères n'est en revanche nullement affecté par ce parti pris, car le second établissement monastique est toujours venu en relais, si l'on peut dire, d'établissements plus « classiques »; or il est réduit à une quinzaine d'attestations, ce qui revient à dire que près de quatre villes sur cinq n'ont pas été touchées par le monachisme. Cette pauvreté d'ensemble serait d'ailleurs plus accusée si l'on prenait en compte des particularités provinciales qui sont ici encore plus marquées qu'en d'autres domaines. Sans doute la hiérarchie est-elle sensiblement la même que sur le tableau II où est recensé l'ensemble des édifices chrétiens, mais le contraste est beaucoup plus patent entre les trois provinces de tête, Aquitaine Première, Viennoise et Aquitaine Seconde, et les quatre autres provinces qui ne comptent à coup sûr qu'un établissement monastique en Novempopulanie et dans les Alpes Maritimes, et aucun dans les deux Narbonnaises. Ce qui ne manque pas de surprendre, au moins pour ces trois dernières provinces, si l'on songe à la multiplicité des premières installations monastiques sur le littoral méditerranéen que nous fait connaître la préface des Conférences XVIII à XXIV de Cassien. Est-ce dû, au moins pour une part, à une carence documentaire ? Peut-être : après tout, le même Cassien atteste du projet qu'avait l'évêque Castor d'Apt de créer un monastère, mais ce monastère est inconnu des sources ultérieures (et donc absent du tableau), sans que l'on sache si cela tient au fait que le projet a avorté, que la fondation est restée trop obscure ou qu'elle a été éphémère.

Mais revenons à la topographie, et plus précisément à la distinction entre établissements monastiques urbains et extra-urbains qui est évidemment essentielle, même si certains monastères extra muros étaient fort proches du rempart, comme celui de Cybard à Angoulême, qui est le seul dans le Midi dont l'archéologie ait retrouvé des traces, d'ailleurs assez ténues (fig. 48) (Boissavit-Camus, 1989). Cette distinction n'est pas des plus faciles à faire cependant, à la fois parce que les sources écrites n'indiquent pas toujours la localisation de fondations qui ont disparu et parce que la topographie de certaines grandes villes, comme Vienne, est encore trop mal connue pendant l'Antiquité tardive pour que l'on puisse trancher sur le statut intra ou extra-urbain de leurs monastères. Dans ces conditions, évaluer à moins d'une dizaine (soit $20 \%$ environ de l'ensemble) le nombre des établissements sûrement installés intra muros n'est qu'une approximation, qui pèche certainement par défaut.

Au moins est-il sûr qu'au sein des rares villes où ces couvents sont attestés - Arles, Bourges, Cahors et Poitiers ils ont certainement contribué à remodeler profondément la topographie, surtout lorsqu'ils avaient l'importance de Saint-Jean d'Arles ou de la fondation royale de Radegonde à Poitiers. Leur impact a été plus important encore que celui des groupes cathédraux, dont l'érection ne faisait qu'ajouter un édifice à la parure monumentale des villes; dans le cas des monastères - et surtout des monastères féminins, dont la clôture était particulièrement stricte - la construction de ces véritables «villes dans la ville » qu'étaient les établissements monastiques a conduit à une réelle déchirure 


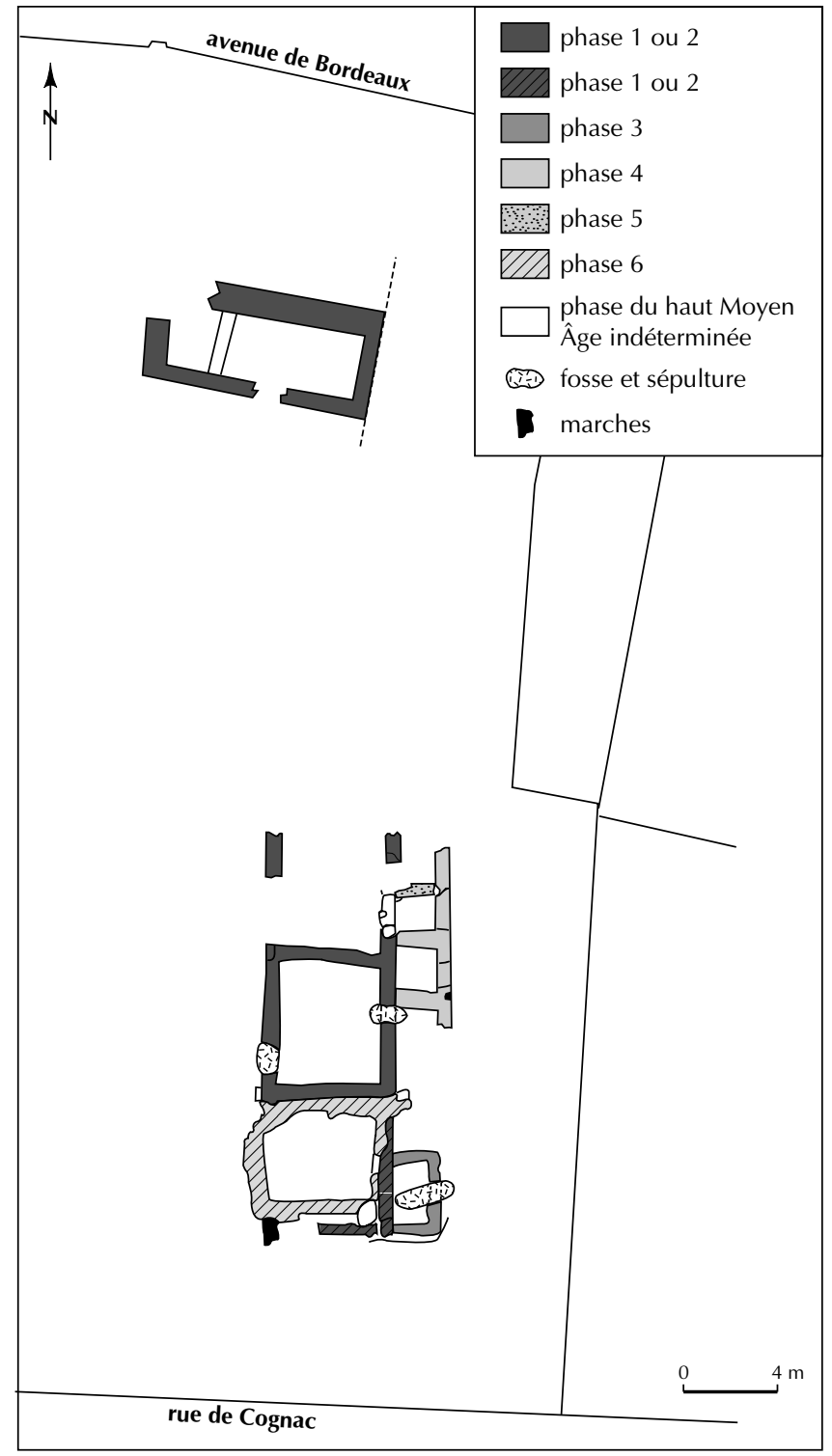

Fig. 48 - Angoulême (Charente). Les vestiges paléochrétiens de l'abbaye Saint-Cybard (dessin P. Mornais et C. Ranche d'après Boissavit-Camus, 1989, p. 43).

du tissu urbain, qui s'est trouvé comme nécrosé à leur emplacement, et le fait que ces couvents, ne fût-ce que par les fonctions d'assistance qu'ils remplissaient, étaient aussi des centres de vie, ne remédiait que modérément à cet état de fait. Il en a été tout autrement hors les murs, où les monastères sont venus au contraire étoffer toute une panoplie de monuments chrétiens dont la floraison pendant l'Antiquité tardive a donné un nouveau visage au paysage du suburbium.

\section{LES ÉDIFICES EXTRA MUROS}

C'est hors les murs, on l'a dit, que l'affirmation d'une topographie chrétienne a été la plus sensible puisque les édifices extra-urbains représentent les deux tiers des monuments chrétiens, groupes épiscopaux exclus, que compte le Midi. Si l'on ajoute qu'à retenir les établissements réputés incertains pour une raison ou une autre, ces édifices sont attestés dans près de quatre villes sur cinq, sans qu'il y ait de province où ils ne soient pas représentés, leur floraison participait donc d'un phénomène très largement partagé.

Cela se comprend, s'agissant pour l'essentiel de monuments dont la création doit tout au culte des saints, qui fut la grande affaire de l'Antiquité tardive, et leur succès à l'attrait que présentait pour une élection de sépulture la proximité des reliques qu'ils abritaient. Ce phénomène de la tumulatio ad sanctos explique que se sont constitués au sein et autour de ces édifices " des cimetières de chrétiens ", mais non encore des cimetières chrétiens au sens où le Moyen Âge a entendu ce mot (Treffort, 1996), nombre de fidèles continuant à être enterrés dans d'autres nécropoles dépourvues de sanctuaires aux côtés de leurs contemporains, de quelque religion qu'ils fussent. L'Église antique n’a jamais réglementé en effet les usages funéraires, de sorte qu'hors des édifices de culte ou de leurs abords, il faut disposer de signes sans équivoque de la foi du défunt avant de décider si une tombe est ou non chrétienne, de simples présomptions, comme le fait qu'elle soit orientée ou dépourvue d'offrandes, ne constituant nullement des preuves, quoi qu'on en dise ou en écrive encore trop souvent.

Comme l'a bien montré la thèse de B. Beaujard, rares sont dans les Gaules jusqu'au tournant des années 400 les témoignages d'une dévotion aux saints (Beaujard, 2000), ce qui explique que, sauf exception, les premiers sanctuaires de martyrs n'apparaissent guère dans le Midi avant le $\mathrm{V}^{\mathrm{e}} \mathrm{s}$. Mais leur diffusion a dès lors été très rapide, comme en témoignent les deux ouvrages complémentaires de Grégoire de Tours, La Gloire des martyrs et La Gloire des confesseurs, grâce auxquels on peut disposer pour la fin du $\mathrm{VI}^{\mathrm{e}} \mathrm{s}$. d'une image, sinon exacte, du moins des plus approchées qui soient du phénomène. Et le processus s'est largement poursuivi aux $\mathrm{VII}^{\mathrm{e}}$ et $\mathrm{VIII}^{\mathrm{e}} \mathrm{s}$. et même au-delà, comme en témoignent les 66 édifices problématiques (le plus souvent pour des raisons chronologiques) qui sont recensés dans le tableau I. 
Qu'il s'agisse de monuments élevés sur des tombes de saints ou de martyrs ou d'édifices qui abritaient des reliques importées, ce sont là autant d'éléments à inscrire dans la topographie funéraire qui avait de tout temps, dans l'Antiquité, marqué les abords des villes. Il s'en faut malheureusement de beaucoup que l'on connaisse précisément les modalités de cette inscription, même lorsqu'elles ont été identifiées par l'archéologie. Les fouilles, qui sont souvent anciennes, n'ont que rarement porté en effet une attention suffisante à la chronologie des tombes; de ce fait, on ignore le plus souvent si les cimetières qui ont été identifiés autour des sanctuaires sont autant d'extensions de nécropoles plus anciennes ou s'ils répondent à des créations ex novo, ce qui n'a pas la même signification topographique. La chose est d'autant plus regrettable que la recherche récente, dans une ville comme Marseille par exemple, tend à revenir sur les estimations trop généreuses qui avaient été faites par le passé à partir de semis de points dus à des découvertes isolées et à abandonner l'image de vastes nécropoles entourant de toutes parts la cité au profit d'un dispositif « en peau de léopard » fait de noyaux cémétériaux plus ou moins distants les uns des autres (Guyon, 2001).

Au sein de ces noyaux, les monuments chrétiens constituaient autant de signes de la foi nouvelle. Ces édifices que les documents écrits, une fois encore, désignent par le terme de «basiliques », étaient des plus variés tant par leur taille que par leur plan. Pour la taille, cela pouvait aller de bâtiments modestes, comme le probable oratoire funéraire de l'évêque Pantagathus de Vienne qui ne dépassait pas, hors tout, une quinzaine de mètres de long, jusqu'à d'amples vaisseaux comme celui de la basilique récemment fouillée rue Malaval à Marseille, dont la largeur est de $15 \mathrm{~m}$, tandis que sa longueur, incomplètement reconnue, dépasse $40 \mathrm{~m}$. Quant au parti architectural, si la plupart des monuments répondaient à un plan basilical plus ou moins ample, certains étaient beaucoup plus originaux : ainsi pour Saint-Victor de Marseille, où l'assise qu'offraient les bancs d'une ancienne carrière a été mise à profit pour élever sur deux niveaux un édifice complexe qui se dressait, tel un signal, face à la ville; ainsi également pour Saint-Laurent de Grenoble, où des mausolées qui étaient originellement étagés sur cet autre site de pente ont été réunis en un tout organique, fermement structuré par un plan cruciforme augmenté d'absides dont le savant jeu de courbes donne à l'ensemble une allure presque baroque (fig. 49). Inutile d'insister : pour une vue d'ensemble de ces sanctuaires extra-urbains, qu'il suffise de renvoyer, sans référence explicite, aux notices des deux premiers volumes des Premiers monuments de la France, auxquelles il conviendra d'ajouter pour Saint-Victor de Marseille les aggiornamenti d'importance que fournit un récent guide du site (Fixot, Pelletier, 2004).

Il s'en faut que l'on connaisse les fondateurs de tous ces édifices. Certains étaient de haute noblesse, comme Pantagathus de Vaison, qui se fit construire au VI ${ }^{\mathrm{e}}$ s. une chapelle funéraire où il plaça des reliques de saint Vincent de Saragosse et de ses compagnons, afin de ne pas avoir «à quémander une concession » auprès d'une autre tombe sainte, comme le dit l'inscription qui conserve la mémoire de l'édifice. D'autres étaient des notables locaux, tel ce Petrus, fils d'Asclépios, qui fit élever à pareille époque dans les Alyscamps d'Arles une église funéraire en l'honneur des Apôtres. Mais la part déterminante est certainement revenue aux évêques, dont on sait depuis les travaux de P. Brown quel rôle majeur ils ont joué dans le développement du culte des saints (Brown, 1984), que l'on ne saurait décidément tenir pour une manifestation de religion populaire (Guyon, 2003). Et c'est en tout cas des évêques que sont venues les premières initiatives: en témoigne Saint-Sernin de Toulouse, qui fournirait une attestation exceptionnellement précoce du développement d'un culte martyrial s'il faut se fier aux allégations de la Passio Saturnini quand elle évoque, au-dessus de la tombe du saint, d'abord la construction d'un modeste édifice - basilicula paruula - par l'évêque Hilaire au milieu du $\mathrm{IV}^{\mathrm{e}} \mathrm{s}$., puis celle d'une basilique beaucoup plus ample et plus belle - pulchra et speciosa - par les soins d'Exupère au début du $\mathrm{V}^{\mathrm{e}} \mathrm{s}$.

La construction de ces édifices répondait en effet à une volonté pastorale, dont on possède également de précoces attestations. Ainsi par ce sermon prononcé à Arles dans les premières décennies $\mathrm{du} \mathrm{V}^{\mathrm{e}} \mathrm{s}$., où l'orateur loue le martyr local Genès pour avoir sanctifié les deux rives du Rhône - et donc les deux quartiers de cette ville double : l'un par son sang, qu'il avait versé rive droite, à Trinquetaille, où l'on vénérait le mûrier sous lequel il avait été exécuté; l'autre par sa tombe qui était rive gauche, aux Alyscamps (fig. 50). Ce souci de sanctifier la topographie des abords de la ville se comprend : il tenait au fait qu'aux yeux des évêques et, plus largement, de leurs contemporains, les reliques des saints constituaient le meilleur rempart de la cité, comme en témoigne pour le VI ${ }^{\mathrm{e}}$ s. l'auteur de la Vita de Maxime de Riez, qui se félicite que la ville soit devenue inexpugnable depuis que le saint évêque repose à ses portes.

Ainsi s'explique que l'on ait parfois tenu à sanctifier le rempart lui-même, comme à Poitiers où un oratoire dédié 


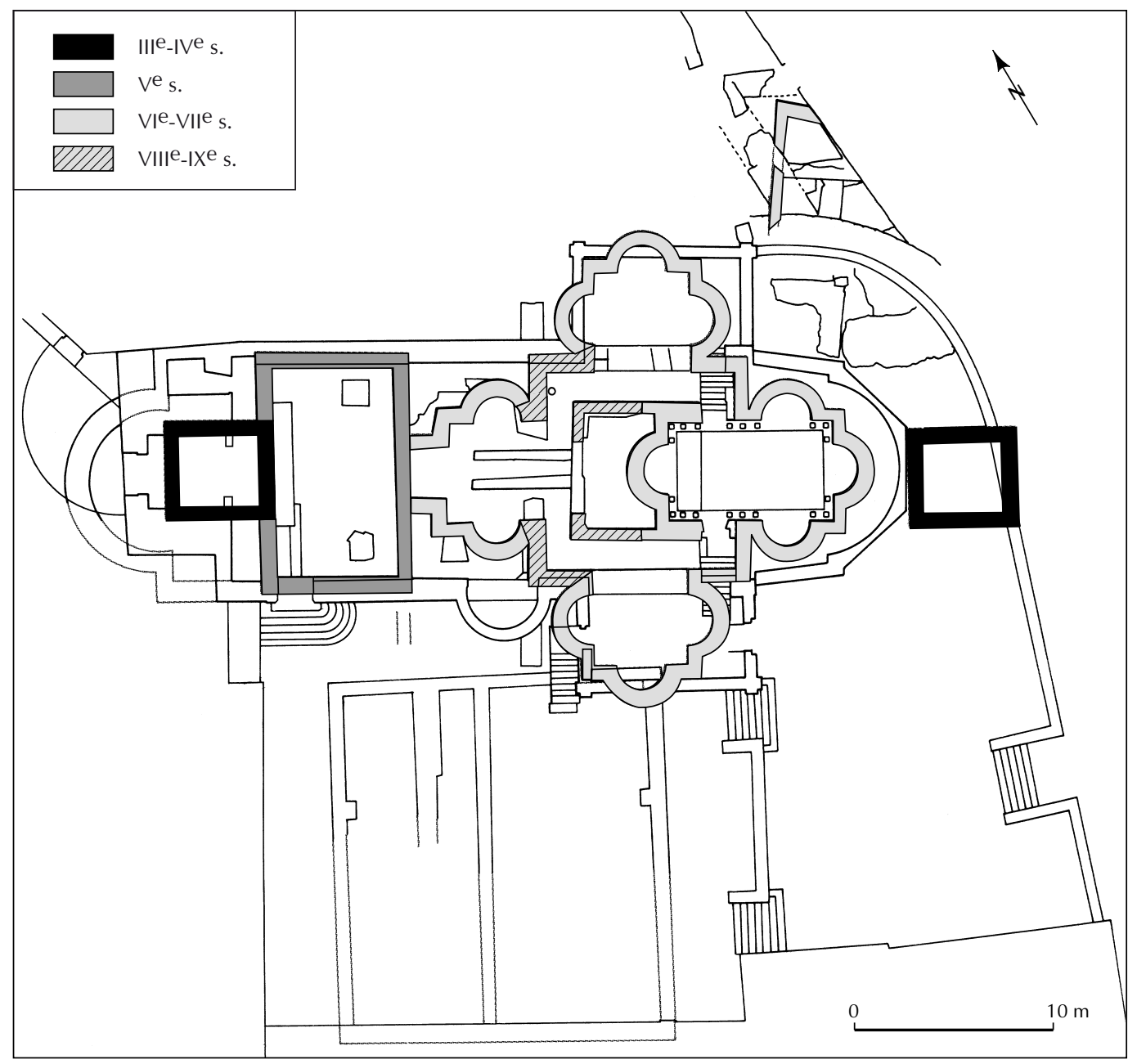

Fig. 49 - Grenoble (Isère). Plan de la basilique Saint-Laurent (dessin Centre d'archéologie historique des musées de Grenoble et de l'Isère, d'après Duval dir., 1995, p. 241).

à saint Michel fut élevé à la porte même de la ville. Ainsi s'explique également que l'on ait multiplié les basiliques alentour de la courtine, comme autant de défenses avancées. Marseille offre de ce point de vue un exemple de choix car dans cette ville où l'on connaissait de longue date l'existence, sur la rive sud du Lacydon, de la basilique Saint-Victor, mais aussi d'autres sanctuaires, certains ou probables, qui en jalonnaient le cours, les recherches récentes permettent d'étendre à l'ensemble des abords de la ville la panoplie de ces fondations funéraires: au sud-est d'abord, dans une nécropole relativement éloignée qui comptait probablement une basilique; à l'est ensuite, où le réexamen du dossier des fouilles de la Bourse permet de supposer l'existence d'une autre basilique à proximité de la porte d'Italie (Guyon, 2001) ; au nord enfin, où les récentes découvertes faites rue Malaval ont livré, auprès de la porte d'Aix, une dernière basilique pourvue de reliques, pour laquelle on renverra dans ce dossier à l'article de M. Moliner (voir infra, p. 131-136) (fig. 51).

Le même phénomène se vérifie, où à peu près, dans cette autre ville majeure qu'était Clermont, où il n'est guère que le sud qui ait été à peu près dépourvu d'établissements funéraires ou de sanctuaires (fig. 52), mais en ce cas, la densité des fondations était telle au nord qu'elle avait donné lieu à la constitution hors les murs d'un véritable «faubourg chrétien », comme l'appelaient les Clermontois eux-mêmes si l'on en croit Grégoire de Tours : "vicus quem christianorum vocant». Une telle appellation n'a rien d'étonnant si l'on songe qu'elle s'applique à un centre si actif de pastorale que l'on y avait - chose exceptionnelle - installé un baptistère et 


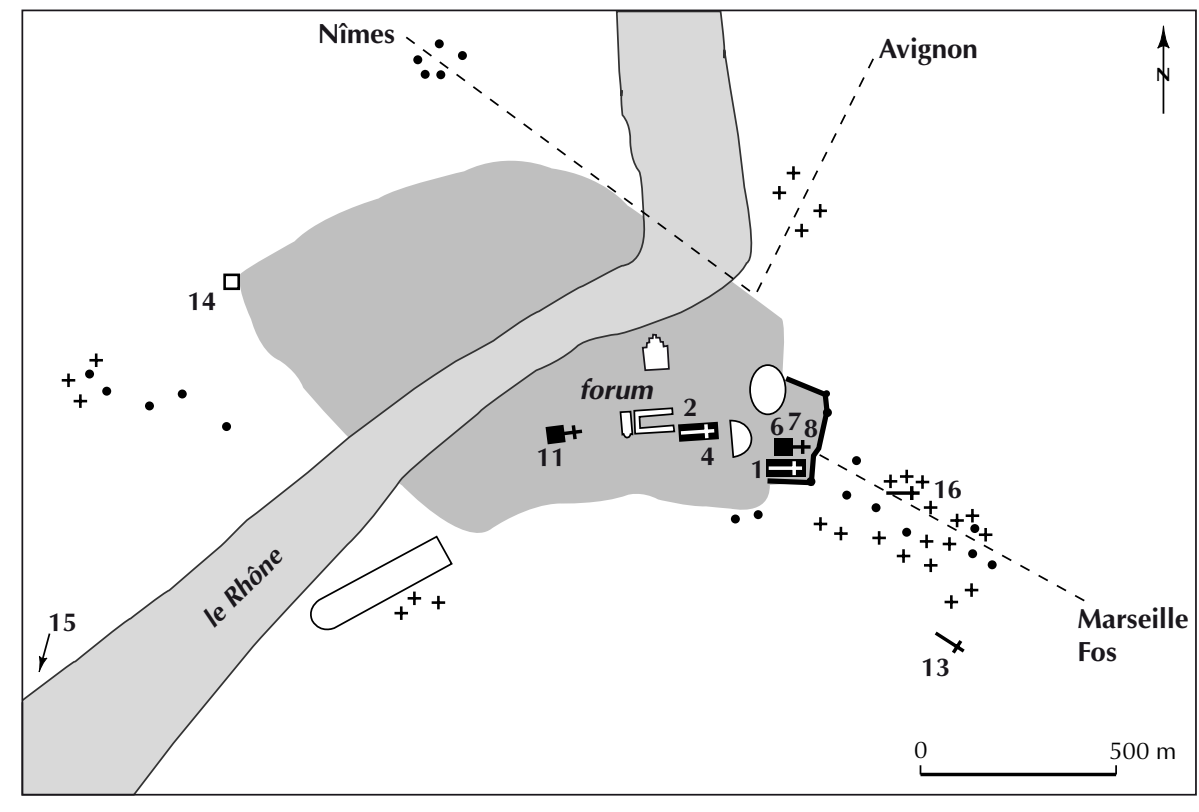

\begin{tabular}{|c|c|c|c|}
\hline$\Psi$ cathédrale & - baptistère & $\because$ nécropole païenne & - - - voie \\
\hline + basilique & $\square \quad$ lieu de culte médiéval & & \\
\hline - monastère & habitat du Haut-Empire & $+_{+}^{+}$nécropole chrétienne & — rempart antique \\
\hline
\end{tabular}

Fig. 50 - Arles (Bouches-du-Rhône). Topographie chrétienne pendant l'Antiquité tardive (les numéros renvoient à la liste des édifices chrétiens de la TCCG, III, 1986b, p. 75) (dessin S. Roucole). Édifices antiques : 1, groupe épiscopal primitif; 2, groupe épiscopal du Ve s. ; 4, résidence épiscopale ; 6-8, monastère féminin de Saint-Jean, Saint-Pierre et Sainte-Croix ; 11, monastère d'hommes fondé par Aurélien ; 13, Saint-Genès aux Alyscamps ; 14, Saint-Genès à la Colonne ; 15, monastère insulaire suburbain ; 16, basilique Saint-Pierre-Saint-Paul.

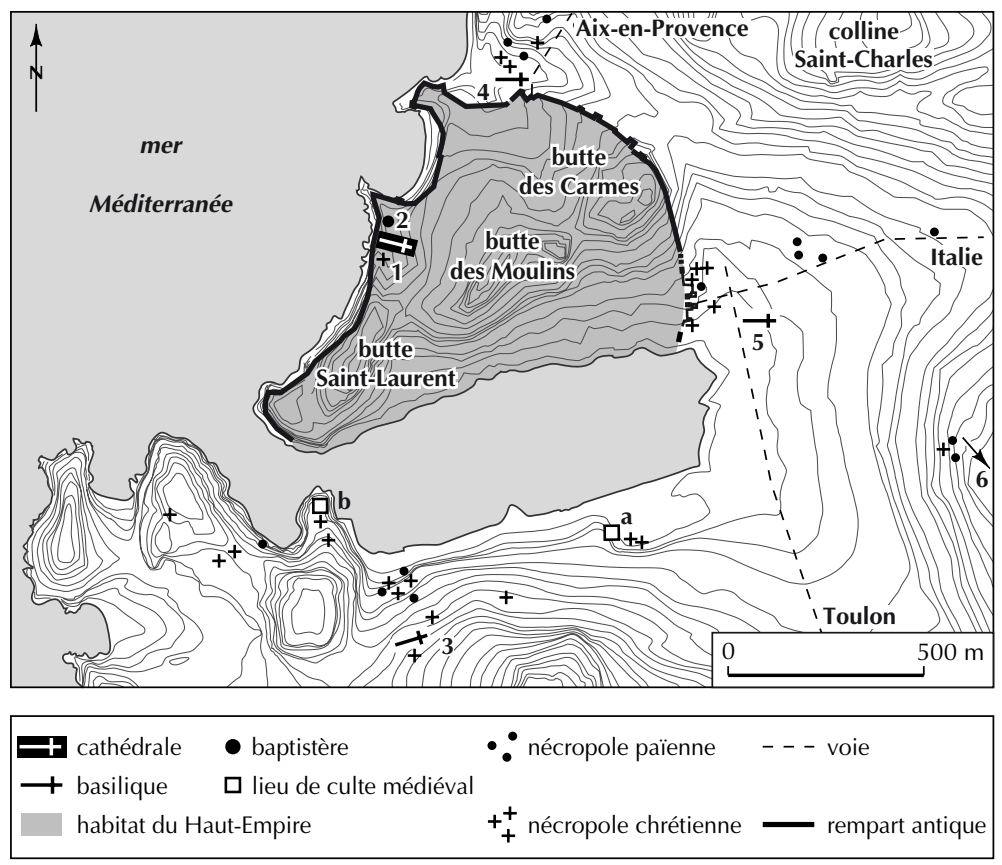

Fig. 51 - Marseille (Bouches-du-Rhône). Topographie chrétienne pendant l'Antiquité tardive (dessin M. Bouiron). Édifices antiques : 1, cathédrale ; 2, baptistère ; 3, Saint-Victor; 4, basilique funéraire de la rue Malaval ; 5, basilique funéraire du site de la Bourse; 6, basilique funéraire du quartier de la Plaine. Édifices de date incertaine : a, Saint-Pierre; b, Saint-Nicolas. 


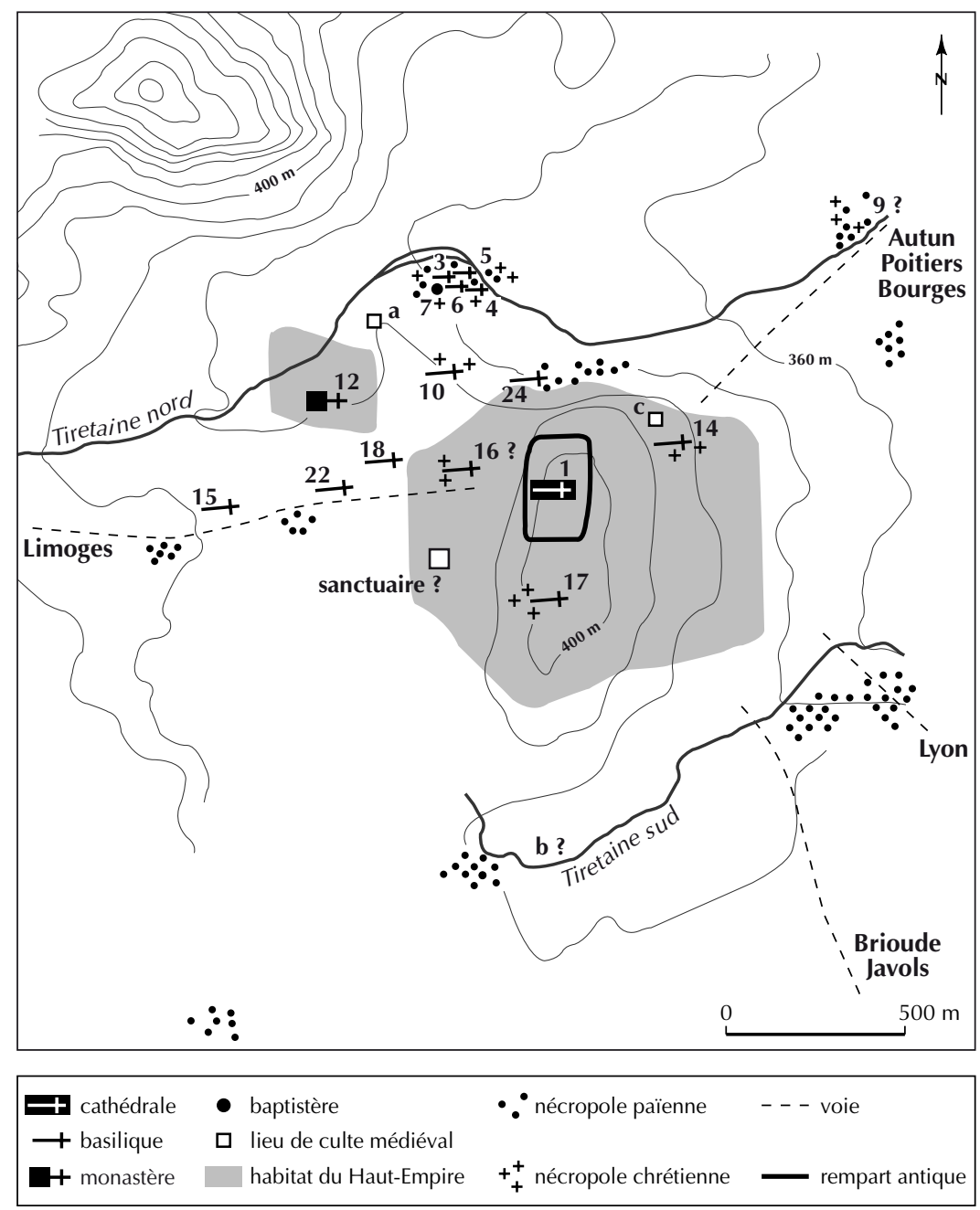

Fig. 52 - Clermont-Ferrand (Puy-de-Dôme). Topographie chrétienne pendant l’Antiquité tardive (les numéros renvoient à la liste des édifices chrétiens de la TCCG, II, 1989a, p. 27) (dessin S. Roucole). Edifices antiques : 1, groupe épiscopal ; 3, Saint-Alyre ; 4, Saint-Vénérand ; 5, Saint-Cassius ; 6, Saint-Antolien ; 7, baptistère Saint-Jean ; 9, Crypta Cantabennensis ; 10, Saint-Étienne ; 12, Saint-Cyr ; 14, Saint-Laurent ; 15, Saint-André ; 16, Saint-Pierre; 17, Saint-Symphorien ; 18, Saint-Adjutor ; 22, Xenodochium ; 24, Saint-Maurice. Édifices de date incertaine : a, Saint-Artémius ; b, Saint-Saturnin ; c, Sainte-Marie.

que des abbayes étaient en outre liées à certaines des basiliques qu'il comptait; tous ces éléments destinés à la desserte spirituelle, mais également matérielle, des fidèles locaux et des pèlerins attirés, souvent de loin, par le culte des reliques étaient bien aptes à fixer un habitat important.

Mais surtout, l'indication fournie par Grégoire de Tours est précieuse en ce sens qu'elle permet d'imaginer l'existence de faubourgs plus ou moins analogues dans des villes qui comptaient de semblables concentrations d'édifices de culte hors les murs : ainsi à Bourges, auprès de SaintOutrille où étaient réunis tous les lieux d'assistance dont l’Église antique était prodigue, matricule, asile et hôpital; ainsi également à Poitiers, aux abords de Saint-Hilaire où se trouvaient de nombreux bâtiments que l'on entrevoit au détour des épisodes de la ténébreuse affaire des moniales de Sainte-Croix en révolte contre leur abbesse, tels que nous les a narrés Grégoire de Tours. Il serait un peu vain d'essayer d'allonger la liste, quand la création de tels faubourgs est probablement restée un phénomène d'exception. Mais le fait qu'ils aient existé ici ou là donne la preuve de la dynamique qu'a créée dans les villes et leurs abords l'émergence d'une topographie chrétienne, au point que l'empreinte en reste encore sensible aujourd'hui dans le paysage. 


\section{L'APPORT DE LA TOPOGRAPHIE CHRÉTIENNE À LA MORPHOGENÈSE DE LA VILLE MÉDIÉVALE ET MODERNE}

Les traces de la topographie chrétienne de l'Antiquité tardive sont particulièrement évidentes dans les villes romaines qui ne sont plus aujourd'hui que des bourgades, comme à Saint-Bertrand-de-Comminges où les éléments qui gardent trace de l'antique Convenae, le village sur la hauteur, le faubourg du Plan à ses pieds et, plus loin, Valcabrère se signalent encore, l'un par la cathédrale qui a succédé à l'ecclesia, l'autre par sa basilique paléochrétienne et le dernier par l'église Saint-Just, lointaine héritière sans doute d'une basilique funéraire antique (fig. 47). Mais ces traces ne sont pas moins apparentes, pour qui sait les voir, dans le paysage de villes qui sont restées importantes : ainsi à Marseille, où la masse de l'abbaye Saint-Victor répond encore à celle de la cathédrale située sur l'autre rive du port, comme le martyrium du saint répondait pendant l'Antiquité tardive à celle du groupe épiscopal.

Le plus important ne tient pas cependant à ces vestiges d'une topographie antique, mais à tout ce que la genèse des villes médiévales et modernes doit au premier établissement chrétien. Ainsi à Clermont-Ferrand, que nous avons évoquée à l'instant: si Saint-Alyre est aujourd'hui le nom d'un quartier de la ville, c'est en souvenir de l'abbaye médiévale qui avait succédé au vicus christianorum de Grégoire de Tours, pérennisant à proximité un habitat qui s'est développé au Moyen Âge. Mais il en est de même pour bien d'autres villes dont le paysage, parfois jusqu'à une époque récente, a été marqué par une semblable dualité entre «bourg» et «cité », qui gardait trace de réalités

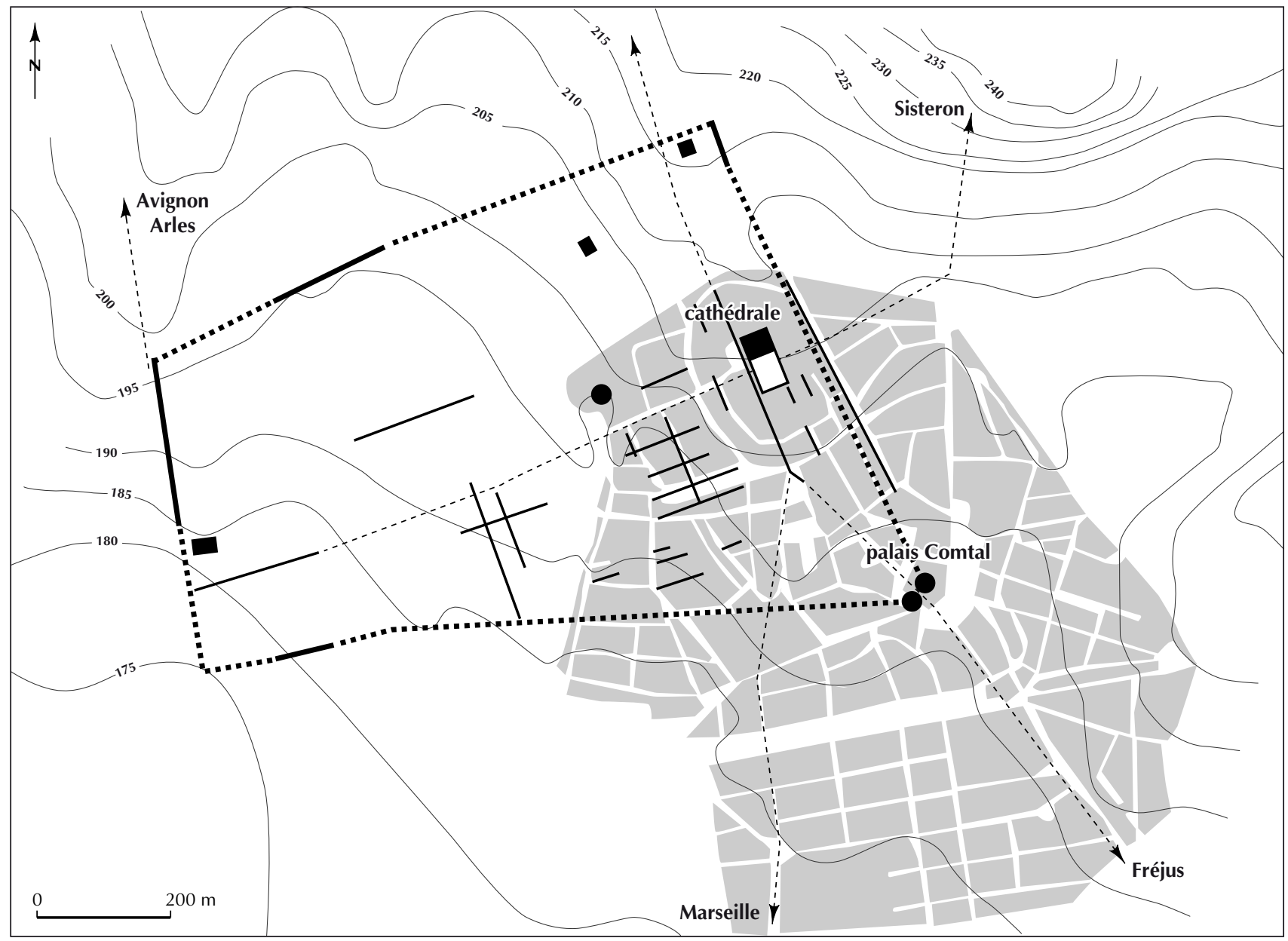

Fig. 53 - Aix-en-Provence (Bouches-du-Rhône) : antique, en pointillés ; moderne, en grisé (dessin V. Gémonet, CNRS, d'après Coste, 1985, p. 23). 
urbaines souvent nées pendant l'Antiquité tardive ou le haut Moyen Âge autour d'établissements chrétiens situés, pour les uns, intra muros et, pour les autres, extra muros. Le phénomène est trop connu pour que l'on s'y attarde; mieux vaut donc s'attacher pour finir au cas d'Aix-enProvence, plus révélateur encore peut-être, parce que plus singulier.

Passé l'étiage du haut Moyen Âge, quand la ville d'Aix renaît à époque romane, ce fut à la faveur de trois noyaux urbains fortifiés qui avaient pris place aux deux extrémités de l'ancienne Aquae Sextiae: le premier à l'ouest, autour de Notre-Dame-de-la-Seds, siège probable de l'ecclesia primitive; les deux autres à l'est, respectivement autour de la seconde cathédrale et du palais comtal qui avait été installé sur les restes de la porte d'Italie (fig. 43). Or si la « ville des Tours ", à l'ouest, a périclité pour disparaître finalement au bas
Moyen Âge, il n'en est pas allé de même pour les deux autres noyaux, auprès desquels des faubourgs se sont rapidement développés, surtout en direction du Midi. Ainsi s'explique qu'Aix à l'époque moderne ait été largement située à l'écart de la ville antique, où étaient situés ces "clos " célébrés par Zola qui ont subsisté aux abords de l'agglomération jusqu'au milieu du $\mathrm{XX}^{\mathrm{e}} \mathrm{s}$. ou peu s'en faut. Une telle translation constitue un cas unique dans le Midi; or elle doit tout aux choix qui furent faits autour des années 500 pour l'établissement de la seconde cathédrale, par laquelle passe l'axe selon lequel s'est effectué le glissement (fig. 53). Où l'on vérifie, une nouvelle fois, l'intérêt que présente l'étude de la topographie chrétienne, non seulement en elle-même, mais surtout comme " marqueur » de l'évolution des villes, aussi bien pendant l'Antiquité tardive qu'aux époques ultérieures. 